\title{
Research Article \\ Contractions of Product Density Operators of Systems of Identical Fermions and Bosons
}

\author{
Wiktor Radzki ${ }^{1,2}$ \\ ${ }^{1}$ Faculty of Mathematics and Computer Science, Nicolaus Copernicus University, ul. Chopina 12/18, \\ 87-100 Torun, Poland \\ 2 School of Mathematics, West Pomeranian University of Technology, Szczecin, al. Piastów 17, \\ 70-310 Szczecin, Poland
}

Correspondence should be addressed to Wiktor Radzki, wiktorradzki@yahoo.com

Received 17 May 2010; Accepted 5 December 2010

Academic Editor: Asao Arai

Copyright (C) 2010 Wiktor Radzki. This is an open access article distributed under the Creative Commons Attribution License, which permits unrestricted use, distribution, and reproduction in any medium, provided the original work is properly cited.

\begin{abstract}
Recurrence and explicit formulae for contractions (partial traces) of antisymmetric and symmetric products of identical trace class operators are derived. Contractions of product density operators of systems of identical fermions and bosons are proved to be asymptotically equivalent to, respectively, antisymmetric and symmetric products of density operators of a single particle, multiplied by a normalization integer. The asymptotic equivalence relation is defined in terms of the thermodynamic limit of expectation values of observables in the states represented by given density operators. For some weaker relation of asymptotic equivalence, concerning the thermodynamic limit of expectation values of product observables, normalized antisymmetric and symmetric products of density operators of a single particle are shown to be equivalent to tensor products of density operators of a single particle.
\end{abstract}

\section{Introduction}

This paper (see also preprint [1]), presenting the results of a part of the author's thesis [2], deals with contractions (partial traces) of antisymmetric and symmetric product density operators representing mixed states of systems of identical noninteracting fermions and bosons, respectively.

If $\mathscr{H}$ is a separable Hilbert space of a single fermion (boson), then the space of the $n$ fermion (resp. $n$-boson) system is the antisymmetric (resp. symmetric) subspace $\mathscr{L}^{\wedge n}$ (resp. $\mathscr{L}^{\vee n}$ ) of $\mathscr{L}^{\otimes n}$. Density operators of $n$-fermion (resp. $n$-boson) systems are identified with those defined on $\mathscr{L}^{\otimes n}$ and concentrated on $\mathscr{L}^{\wedge n}$ (resp. $\left.\mathscr{L}^{\vee n}\right)$. 
Recall that the expectation value of an observable represented by a bounded selfadjoint operator $B$ on given Hilbert space in a state described by a density operator $\rho$ equals $\operatorname{Tr} B \rho$. If $B$ is an unbounded self-adjoint operator on a dense subspace of given Hilbert space, instead of $B$ one can consider its spectral measure $E_{B}(\Delta)$ (which is a bounded operator) of a Borel subset $\Delta$ of the spectrum of $B$. Then $\operatorname{Tr} E_{B}(\Delta) \rho$ is the probability that the result of the measurement of the observable in question belongs to $\Delta[3]$.

$k$-particle observables of $n$-fermion and $n$-boson systems $(k<n)$ are represented, respectively, by operators of the form

$$
\hat{\Gamma_{k}^{n}} B=A_{\mathscr{d}}^{(n)}\left(B \otimes I^{\otimes(n-k)}\right) A_{\mathscr{d}}^{(n)}, \quad \stackrel{\vee}{\Gamma_{k}^{n}} B=S_{\mathscr{d}}^{(n)}\left(B \otimes I^{\otimes(n-k)}\right) S_{\mathscr{d}}^{(n)}
$$

(multiplied by $\left(\begin{array}{c}n \\ k\end{array}\right)$ ), where $A_{\mathscr{l}}^{(n)}$ and $S_{\mathscr{\ell}}^{(n)}$ are projectors of $\mathscr{L}^{\otimes n}$ onto $\mathscr{L}^{\wedge n}$ and $\mathscr{L}^{\vee n}$, respectively, $I$ is the identity operator on $\mathscr{d}$ and $B$ is a self-adjoint operator on $\mathscr{l}^{\otimes k}$ (see [4]). Operators (1.1) are called antisymmetric and symmetric expansions of $B$. In view of the earlier remark it is assumed that $B$ is bounded. The expectation values of observables represented by $\hat{\Gamma}_{k}^{n} B$ and $\stackrel{\vee}{\Gamma_{k}^{n}} B$ in states represented by $n$-fermion and $n$-boson density operators $K$ and $G$, respectively, can be expressed as

$$
\operatorname{Tr} K \hat{\Gamma_{k}^{n}} B=\operatorname{Tr} B \mathrm{~L}_{n}^{k} K, \quad \operatorname{Tr} G \stackrel{\vee}{\Gamma_{k}^{n}} B=\operatorname{Tr} B \mathrm{~L}_{n}^{k} G
$$

(see [4, equations (1.7), (3.19)]), where $k$-particle density operators $\mathrm{L}_{n}^{k} K$ and $\mathrm{L}_{n}^{k} G$ are $(n, k)$ contractions of $K$ and $G$ (see Definition 2.1), called also reduced density operators. Such operators were investigated by Coleman [5], Garrod and Percus [6], and Kummer [4] (see also, e.g., [7-9] and references therein). A presentation of the basic ideas concerning reduced density operators and their applications can be found in [10].

In the present paper particular interest is taken in the case when $K$ and $G$ are product density operators, that is, they are of the form

$$
K=\frac{1}{\operatorname{Tr} \rho^{\wedge n}} \rho^{\wedge n}, \quad G=\frac{1}{\operatorname{Tr} \rho^{\vee n}} \rho^{\vee n},
$$

where $\rho^{\wedge n}=A_{\mathscr{d}}^{(n)} \rho^{\otimes n} A_{\mathscr{l}}^{(n)}, \rho^{\vee n}=S_{\mathscr{d}}^{(n)} \rho^{\otimes n} S_{\mathscr{l}}^{(n)}$, and $\rho$ is a density operator of a single fermion or boson, respectively. The first objective of this paper is to find the recurrence and explicit formulae for $\mathrm{L}_{n}^{k} K$ and $\mathrm{L}_{n}^{k} G$ for $K$ and $G$ being, respectively, antisymmetric and symmetric products of identical trace class operators, including operators (1.3). The explicit form of the operators $L_{n}^{k} K$ and $L_{n}^{k} G$ proves to be quite complex. However, they can be replaced by operators with simpler structure if only the limiting values of expectations (1.2), in the sense of the thermodynamic limit, are of interest. The second objective of this paper is to find that simpler forms of contractions $\mathrm{L}_{n}^{k} K$ and $\mathrm{L}_{n}^{k} G$ for product density operators (1.3), equivalent to the complete expressions in the thermodynamic limit.

The problems described above have been solved for $k=1,2$ by Kossakowski and Maćkowiak [11], and Maćkowiak [12]. The formulae they derived were exploited in calculations of the free energy density of large interacting $n$-fermion and $n$-boson systems $[11,12]$, as well as in the perturbation expansion of the free energy density for the $M$-impurity 
Kondo Hamiltonian [13]. In the case of investigation of many-particle interactions of higher order [14-17], or higher order perturbation expansion terms of the free energy density, the expressions for $\left(\operatorname{Tr} \rho^{\wedge n}\right)^{-1} \mathrm{~L}_{n}^{k} \rho^{\wedge n}$ and $\left(\operatorname{Tr} \rho^{\vee n}\right)^{-1} \mathrm{~L}_{n}^{k} \rho^{\vee n}$ with $k \geq 3$ can be used in the canonical and grand canonical ensemble approach, which is the physical motivation for the present paper.

The main results of this paper are Theorems 3.1, 3.4, 4.9, and 4.14.

\section{Preliminaries}

In this section notation and terminology are set up.

\subsection{Basic Notation}

Let $(\mathscr{L},\langle\cdot, \cdot\rangle)$ be a separable Hilbert space over $\mathbb{C}$ or $\mathbb{R}$. The following notation is used in the sequel.

$I$ : the identity operator on $\mathscr{H}$,

$B(\mathscr{l})$ : the space of bounded linear operators on $\mathscr{H}$ with the operator norm $\|\cdot\|$,

$\tau(\mathscr{L})$ : the space of trace class operators on $\mathscr{H}$ with the trace norm $\operatorname{Tr}|\cdot|$,

$\mathfrak{B}^{*}(\mathscr{L})$ : the space of bounded self-adjoint operators on $\mathscr{L}$,

$B_{\geq 0}^{*}(\mathscr{\ell})$ : the set of nonnegative definite bounded self-adjoint operators on $\mathscr{\ell}$,

$\Phi(\mathscr{L})$ : the set of density operators (matrices) on $\mathscr{H}$, that is,

$$
\Phi(\mathscr{\ell})=\left\{D \in \tau(\mathscr{\ell}) \mid D=D^{*}, D \geq 0, \operatorname{Tr} D=1\right\} .
$$

Set $\mathscr{H}^{\otimes n}=\underbrace{\mathscr{H} \otimes \cdots \otimes \mathscr{H}}_{n}$ and denote by $S_{n}$ the group of permutations of the set $\{1, \ldots, n\}$. Let $A_{\mathscr{l}}^{(n)}, S_{\mathscr{d}}^{(n)} \in \mathcal{B}\left(\mathscr{L}^{\otimes n}\right)$ be the projectors such that

$$
\begin{gathered}
A_{\mathscr{d}}^{(n)}\left(\psi_{1} \otimes \cdots \otimes \psi_{n}\right)=\frac{1}{n !} \sum_{\pi \in S_{n}} \operatorname{sgn} \pi \psi_{\pi(1)} \otimes \cdots \otimes \psi_{\pi(n)}, \\
S_{\mathscr{d}}^{(n)}\left(\psi_{1} \otimes \cdots \otimes \psi_{n}\right)=\frac{1}{n !} \sum_{\pi \in S_{n}} \psi_{\pi(1)} \otimes \cdots \otimes \psi_{\pi(n)}
\end{gathered}
$$

for every $\psi_{1}, \ldots, \psi_{n} \in \mathscr{l}$. The closed linear subspaces $\mathscr{L}^{\wedge n}=A_{\mathscr{\ell}}^{(n)} \mathscr{\ell}^{\otimes n}$ and $\mathscr{L}^{\vee n}=S_{\mathscr{\ell}}^{(n)} \mathscr{\ell}^{\otimes n}$ of $\mathscr{L}^{\otimes n}$ are called the antisymmetric and symmetric subspace, respectively.

The antisymmetric and symmetric product of operators $B \in \mathcal{B}\left(\mathscr{L}^{\otimes k}\right), C \in \mathcal{B}\left(\mathscr{L}^{\otimes m}\right)$ are defined as $B \wedge C=A_{\mathscr{d}}^{(k+m)}(B \otimes C) A_{\mathscr{d}}^{(k+m)}$ and $B \vee C=S_{\mathscr{l}}^{(k+m)}(B \otimes C) S_{\mathscr{d}}^{(k+m)}$, respectively. It is assumed $B^{\wedge n}=\underbrace{B \wedge \cdots \wedge B}_{n}, B^{\vee n}=\underbrace{B \vee \ldots \vee B}_{n}$, and $B^{\wedge 1}=B^{\vee 1}=B$. Clearly, if $B \in B(\mathscr{L})$ then $B^{\wedge n}=A_{\mathscr{l}}^{(n)} B^{\otimes n}=B^{\otimes n} A_{\mathscr{l}}^{(n)}, B^{\vee n}=S_{\mathscr{d}}^{(n)} B^{\otimes n}=B^{\otimes n} S_{\mathscr{d}}^{(n)}$, and if $B \in B_{\geq 0}^{*}(\mathscr{l})$ then $B^{\wedge n}, B^{\vee n} \in$ $B_{\geq 0}^{*}\left(\mathscr{\ell}^{\otimes n}\right)$. 
Set $\mathbb{R}_{+}=[0,+\infty)$ and $\overline{\mathbb{R}}_{+}=\mathbb{R}_{+} \cup\{+\infty\}$. The product of measures $\mu, \mu_{1}$ is denoted by $\mu \otimes \mu_{1}$ and $\mu^{\otimes n}$ stands for $\underbrace{\mu \otimes \cdots \otimes \mu}_{n}$. In subsequent sections use is made of product integral kernels, described in the appendix.

\subsection{Contractions of Operators}

The definition and basic properties of contractions of operators are now recalled for the reader's convenience. They were studied in [4-7]. A discussion of properties of reduced density operators can also be found in [10].

Let $\mathscr{H}$ be a separable Hilbert space over the field $\mathbb{K}=\mathbb{C}$ or $\mathbb{R}$.

Definition 2.1. Let $k, n \in \mathbb{N}, k<n$, and $K \in \tau\left(\mathscr{\ell}^{\otimes n}\right)$. Then the $(n, k)$-contraction of $K$ is the operator $\mathrm{L}_{n}^{k} K \in \tau\left(\mathscr{\ell}^{\otimes k}\right)$ such that

$$
\forall_{C \in \mathcal{B}\left(\mathscr{H}^{\otimes k}\right)}: \operatorname{Tr}_{\mathscr{H}^{\otimes n}}\left(C \otimes I^{\otimes(n-k)}\right) K=\operatorname{Tr}_{\mathscr{H}^{\otimes k}} C \mathrm{~L}_{n}^{k} K
$$

It is also assumed $\mathrm{L}_{n}^{n} \mathrm{~K}=K$.

Remark 2.2. The operator $\mathrm{L}_{n}^{k} K$ always exists and is defined uniquely by (2.3). $\mathrm{L}_{n}^{k} K$ is a partial trace of $K$ with respect to the component $\mathscr{L}^{\otimes(n-k)}$ of $\mathscr{L}^{\otimes n}=\mathscr{L}^{\otimes k} \otimes \mathscr{L}^{\otimes(n-k)}$. If $\mathscr{L}^{\prime}=\mathscr{L}_{Y}:=$ $L^{2}(Y, \mu)$, where the measure $\mu$ is separable and $\sigma$-finite, and $\mathcal{K}$ is a product integral kernel of $K$ (see the appendix) then $\mathrm{L}_{n}^{k} K$ has an integral kernel $\mathcal{K}_{0}$ given by formula (A.4), according to Lemma A.5 and Corollary A.6.

Under the assumptions of Definition 2.1 one has $\operatorname{Tr}_{\mathscr{L}^{\otimes k}} \mathrm{~L}_{n}^{k} K=\operatorname{Tr}_{\mathscr{\ell}^{8 n}} K$, and if $p \in \mathbb{N}$, $k<p<n$, then $\mathrm{L}_{p}^{k}\left(\mathrm{~L}_{n}^{p} K\right)=\mathrm{L}_{n}^{k} K$. Moreover, if $K \in \mathcal{B}^{*}\left(\mathscr{\ell}^{\otimes n}\right)$ then $\mathrm{L}_{n}^{k} K \in \mathcal{B}^{*}\left(\mathscr{\ell}^{\otimes k}\right)$, and if $K \in \mathbb{B}_{\geq 0}^{*}\left(\mathscr{\ell}^{\otimes n}\right)$ then $\mathrm{L}_{n}^{k} K \in \mathbb{B}_{\geq 0}^{*}\left(\mathscr{\ell}^{\otimes k}\right)$.

Contractions of density operators are called reduced density operators. Contractions preserve the Fermi and the Bose-Einstein statistics of the contracted operator, that is, for $K \in A_{\mathscr{l}}^{(n)} \tau\left(\mathscr{L}^{\otimes n}\right) A_{\mathscr{d}}^{(n)}$ and $G \in S_{\mathscr{d}}^{(n)} \tau\left(\mathscr{L}^{\otimes n}\right) S_{\mathscr{l}}^{(n)}$ one has $\mathrm{L}_{n}^{k} K \in A_{\mathscr{d}}^{(k)} \tau\left(\mathscr{L}^{\otimes k}\right) A_{\mathscr{l}}^{(k)}$ and $\mathrm{L}_{n}^{k} G \in$ $S_{\mathscr{\ell}}^{(k)} \tau\left(\mathscr{L}^{\otimes k}\right) S_{\mathscr{d}}^{(k)}$. For such $K$ and $G(1.2)$ hold.

The following theorem is a part of Coleman's theorem $[4,5]$.

Theorem 2.3. Let $n \in \mathbb{N}, n \geq 2$. For every (n-fermion) density operator $D \in \Phi\left(\mathscr{L}^{\otimes n}\right), D=$ $A_{\mathscr{l}}^{(n)} D A_{\mathscr{l}}^{(n)}$, one has $\left\|L_{n}^{1} D\right\| \leq(1 / n)\|D\|$.

\section{Recurrence and Explicit Formulae for Contractions of Products of Trace Class Operators}

In this section recurrence and explicit formulae for contractions of antisymmetric and symmetric powers of single particle operators are derived.

In the whole section use is made of the Hilbert space $\mathfrak{h}_{Y}:=L^{2}(Y, \mu)$ over the field $\mathbb{K}=\mathbb{C}$ or $\mathbb{R}$, where the measure $\mu$ is separable and $\sigma$-finite. 
International Journal of Mathematics and Mathematical Sciences

The following theorem in the case of $k=1,2$ was proved in [11, 12].

Theorem 3.1 (Recurrence formulae). Let $\rho \in \tau\left(\mathscr{L}_{Y}\right)$. If $k, n \in \mathbb{N}, 1<k<n$, then

$$
\begin{aligned}
\left(\begin{array}{l}
n \\
k
\end{array}\right) \mathrm{L}_{n}^{k} \rho^{\wedge n}= & \left(\begin{array}{c}
n-1 \\
k-1
\end{array}\right)\left(\mathrm{L}_{n-1}^{k-1} \rho^{\wedge(n-1)}\right) \wedge \rho \\
& -\left(\begin{array}{c}
n-1 \\
k
\end{array}\right)\left(\mathrm{L}_{n-1}^{k} \rho^{\wedge(n-1)}\right)\left(I^{\otimes(k-1)} \otimes \rho\right) A_{\mathscr{d}_{\ell^{\prime}}}^{(k)} \\
\left(\begin{array}{l}
n \\
k
\end{array}\right) \mathrm{L}_{n}^{k} \rho^{\vee n}= & \left(\begin{array}{c}
n-1 \\
k-1
\end{array}\right)\left(\mathrm{L}_{n-1}^{k-1} \rho^{\vee(n-1)}\right) \vee \rho \\
& +\left(\begin{array}{c}
n-1 \\
k
\end{array}\right)\left(\mathrm{L}_{n-1}^{k} \rho^{\vee(n-1)}\right)\left(I^{\otimes(k-1)} \otimes \rho\right) S_{\mathscr{d}_{\gamma^{\prime}}}^{(k)}
\end{aligned}
$$

and if $n \in \mathbb{N}, n \geq 2$, then

$$
\begin{aligned}
& n \mathrm{~L}_{n}^{1} \rho^{\wedge n}=\left(\operatorname{Tr} \rho^{\wedge(n-1)}\right) \rho-(n-1)\left(\mathrm{L}_{n-1}^{1} \rho^{\wedge(n-1)}\right) \rho, \\
& n \mathrm{~L}_{n}^{1} \rho^{\vee n}=\left(\operatorname{Tr} \rho^{\vee(n-1)}\right) \rho+(n-1)\left(\mathrm{L}_{n-1}^{1} \rho^{\vee(n-1)}\right) \rho .
\end{aligned}
$$

Proof. Let $\rho: Y \times Y \rightarrow \mathbb{K}$ be a product integral kernel of $\rho$. For every $m \in \mathbb{N}$ define the mapping $\varrho^{\wedge m}: Y^{m} \times Y^{m} \rightarrow \mathbb{K}$ by the formula

$$
\rho^{\wedge m}\left(\begin{array}{l}
x_{1}, \ldots, x_{m} \\
y_{1}, \ldots, y_{m}
\end{array}\right)=\operatorname{det}\left[\begin{array}{ccc}
\rho\left(x_{1}, y_{1}\right) & \cdots & \rho\left(x_{1}, y_{m}\right) \\
\vdots & \cdots & \vdots \\
\rho\left(x_{m}, y_{1}\right) & \cdots & \rho\left(x_{m}, y_{m}\right)
\end{array}\right]
$$

Then the mapping $\mathcal{K}: Y^{n} \times Y^{n} \rightarrow \mathbb{K}$ given by

$$
\mathcal{K}\left(x_{1}, \ldots, x_{n}, y_{1}, \ldots, y_{n}\right)=\frac{1}{n !} \rho^{\wedge n}\left(\begin{array}{c}
x_{1}, \ldots, x_{n} \\
y_{1}, \ldots, y_{n}
\end{array}\right)
$$

is a product integral kernel of $\rho^{\wedge n}=A_{\mathscr{d}_{Y}}^{(n)} \rho^{\otimes n}$. 
Equation (3.1) will be first proved for $n>k+1$. In view of Remark 2.2, an integral kernel $\mathcal{L}: Y^{k} \times Y^{k} \rightarrow \mathbb{K}$ of $\left(\begin{array}{c}n \\ k\end{array}\right) \mathrm{L}_{n}^{k} \rho^{\wedge n}$ can be given by

$$
\mathcal{L}\left(x^{\prime}, y^{\prime}\right)=\left(\begin{array}{l}
n \\
k
\end{array}\right) \int_{Y^{n-k}} \mathcal{K}\left(x^{\prime}, x^{\prime \prime}, y^{\prime}, x^{\prime \prime}\right) \mathrm{d} \mu^{\otimes(n-k)}\left(x^{\prime \prime}\right)
$$

for $\mu^{\otimes 2 k}$-a.a. $\left(x^{\prime}, y^{\prime}\right) \in Y^{k} \times Y^{k}$. Performing $k$ ! permutations of the first $k$ rows and $k$ ! permutations of the first $k$ columns of the determinant defining $\mathcal{K}$ and expanding that determinant with respect to the $k$ th column one obtains

$$
\begin{aligned}
\mathcal{L}\left(x_{1}, \ldots, x_{k}, y_{1}, \ldots, y_{k}\right) \\
=\left(\begin{array}{l}
n \\
k
\end{array}\right) \frac{1}{n !} \frac{1}{(k !)^{2}} \sum_{\pi, \sigma \in S_{k}} \operatorname{sgn} \pi \operatorname{sgn} \sigma \sum_{j=1}^{k}(-1)^{k+j} \\
\quad \times \int_{Y^{n-k}} \rho\left(x_{\pi(j)}, y_{\sigma(k)}\right) \cdot \rho^{\wedge(n-1)}\left(\begin{array}{c}
x_{\pi(1)}, \ldots, x_{\pi(j-1)}, x_{\pi(j+1)}, \ldots, x_{\pi(k)}, x_{k+1}, \ldots, x_{n} \\
y_{\sigma(1)}, \ldots, y_{\sigma(k-1)}, x_{k+1}, \ldots, x_{n}
\end{array}\right) \\
\quad \times \mathrm{d} \mu^{\otimes(n-k)}\left(x_{k+1}, \ldots, x_{n}\right)+\left(\begin{array}{c}
n \\
k
\end{array}\right) \frac{1}{n !} \frac{1}{(k !)^{2}} \sum_{\pi, \sigma \in S_{k}} \operatorname{sgn} \pi \operatorname{sgn} \sigma \sum_{j=k+1}^{n}(-1)^{k+j} \\
\quad \times \int_{Y^{n-k}} \rho\left(x_{j}, y_{\sigma(k)}\right) \cdot \rho^{\wedge(n-1)}\left(\begin{array}{c}
x_{\pi(1)}, \ldots, x_{\pi(k)}, x_{k+1}, \ldots, x_{j-1}, x_{j+1}, \ldots, x_{n} \\
y_{\sigma(1)}, \ldots, y_{\sigma(k-1)}, x_{k+1}, \ldots, x_{n}
\end{array}\right) \\
\quad \times \mathrm{d} \mu^{\otimes(n-k)}\left(x_{k+1}, \ldots, x_{n}\right) .
\end{aligned}
$$

Consider the first term on the r.h.s. of (3.8). In all summands of $\sum_{j=1}^{k}$ except the last one the $(k-1)$ th row of the determinant (containing the variable $\left.x_{\pi(k)}\right)$ can be shifted into the $j$ th position, changing thereby the sign of the determinant by $(-1)^{(k-2)-(j-1)}=(-1)^{-k-j+1}$. Then the first term of sum (3.8) assumes the form

$$
\begin{aligned}
& \left(\begin{array}{l}
n \\
k
\end{array}\right) \frac{1}{n !} \frac{1}{(k !)^{2}} \sum_{\pi, \sigma \in S_{k}} \operatorname{sgn} \pi \operatorname{sgn} \sigma \sum_{j=1}^{k-1}(-1)^{k+j}(-1)^{-k-j+1} \\
& \times \int_{Y^{n-k}} \rho\left(x_{\pi(j)}, y_{\sigma(k)}\right) \cdot \rho^{\wedge(n-1)}\left(\begin{array}{c}
x_{\pi(1)}, \ldots, x_{\pi(j-1)}, x_{\pi(k)}, x_{\pi(j+1)}, \ldots, x_{\pi(k-1)}, x_{k+1}, \ldots, x_{n} \\
y_{\sigma(1)}, \ldots, y_{\sigma(k-1)}, x_{k+1}, \ldots, x_{n}
\end{array}\right)
\end{aligned}
$$




$$
\begin{aligned}
& \times \mathrm{d} \mu^{\otimes(n-k)}\left(x_{k+1}, \ldots, x_{n}\right)+\left(\begin{array}{l}
n \\
k
\end{array}\right) \frac{1}{n !} \frac{1}{(k !)^{2}} \sum_{\pi, \sigma \in S_{k}} \operatorname{sgn} \pi \operatorname{sgn} \sigma(-1)^{k+k} \\
& \times \int_{\Upsilon^{n-k}} \rho\left(x_{\pi(k)}, y_{\sigma(k)}\right) \cdot \rho^{\wedge(n-1)}\left(\begin{array}{c}
x_{\pi(1)}, \ldots, x_{\pi(k-1)}, x_{k+1}, \ldots, x_{n} \\
y_{\sigma(1)}, \ldots, y_{\sigma(k-1)}, x_{k+1}, \ldots, x_{n}
\end{array}\right) \mathrm{d} \mu^{\otimes(n-k)}\left(x_{k+1}, \ldots, x_{n}\right) .
\end{aligned}
$$

Let $T_{j k} \in S_{k}$ denote the transposition $j \leftrightarrow k$ for $j<k$ (then $\left.(-1)^{k+j}(-1)^{-k-j+1}=(-1)=\operatorname{sgn} T_{j k}\right)$ and the identity permutation for $j=k$ (with $\operatorname{sgn} T_{k k}=1$ ). Expression (3.9) can be written as

$$
\begin{aligned}
\sum_{j=1}^{k}\left(\begin{array}{l}
n \\
k
\end{array}\right) \frac{1}{n !} \frac{1}{(k !)^{2}} \sum_{\pi, \sigma \in S_{k}}\left(\operatorname{sgn} \pi \operatorname{sgn} T_{j k}\right) \operatorname{sgn} \sigma \\
\quad \times \int_{Y^{n-k}} \rho\left(x_{\left(\pi \circ T_{j k}\right)(k)}, y_{\sigma(k)}\right) \cdot \rho^{\wedge(n-1)}\left(\begin{array}{c}
x_{\left(\pi \circ T_{j k}\right)(1)}, \ldots, x_{\left(\pi \circ T_{j k}\right)(k-1)}, x_{k+1}, \ldots, x_{n} \\
y_{\sigma(1)}, \ldots, y_{\sigma(k-1)}, x_{k+1}, \ldots, x_{n}
\end{array}\right) \\
\quad \times \mathrm{d} \mu^{\otimes(n-k)}\left(x_{k+1}, \ldots, x_{n}\right) \\
=\left(\begin{array}{c}
n-1 \\
k-1
\end{array}\right) \frac{1}{(k !)^{2}} \sum_{\tau, \sigma \in S_{k}} \operatorname{sgn} \tau \operatorname{sgn} \sigma \rho\left(x_{\tau(k)}, y_{\sigma(k)}\right) \\
\quad \times \int_{Y^{n-k}} \frac{1}{(n-1) !} \cdot \rho^{\wedge(n-1)}\left(\begin{array}{c}
x_{\tau(1)}, \ldots, x_{\tau(k-1)}, x_{k+1}, \ldots, x_{n} \\
y_{\sigma(1)}, \ldots, y_{\sigma(k-1)}, x_{k+1}, \ldots, x_{n}
\end{array}\right) \mathrm{d} \mu^{\otimes(n-k)}\left(x_{k+1}, \ldots, x_{n}\right) .
\end{aligned}
$$

The function $p_{1}: Y^{k} \times Y^{k} \rightarrow \mathbb{K}$, such that $p_{1}\left(x_{1}, \ldots, x_{k}, y_{1}, \ldots, y_{k}\right)$ is $\mu^{\otimes 2 k}$-a.e. equal to expression (3.10), is an integral kernel of the operator

$$
\left(\begin{array}{l}
n-1 \\
k-1
\end{array}\right)\left(\mathrm{L}_{n-1}^{k-1} \rho^{\wedge(n-1)}\right) \wedge \rho,
$$

which appears on the r.h.s. of (3.1).

Consider now the second term of the sum on the r.h.s. of (3.8). One can change the indices of the integral variables $x_{k+1}, \ldots, x_{j}$ in all summands of $\sum_{j=k+1}^{n}$ except the first one, according to the rule $x_{j} \rightarrow x_{k+1} \rightarrow x_{k+2} \rightarrow \cdots \rightarrow x_{j}$ for the $j$ th summand, and simultaneously change the order of the columns of the determinant inversely (which changes 
the sign by $\left.(-1)^{(j-1)-k}=(-1)^{(k+1)-j}\right)$. The resulting sum $\sum_{j=k+1}^{n}$ then contains $n-k$ terms identical to the one with $j=k+1$. Thus the second term of sum (3.8) equals

$$
\begin{aligned}
&-(n-k)\left(\begin{array}{c}
n \\
k
\end{array}\right) \frac{1}{n !} \frac{1}{(k !)^{2}} \sum_{\pi, \sigma \in S_{k}} \operatorname{sgn} \pi \operatorname{sgn} \sigma \\
& \times \int_{Y^{n-k}} \rho\left(x_{k+1}, y_{\sigma(k)}\right) \cdot \rho^{\wedge(n-1)}\left(\begin{array}{c}
x_{\pi(1)}, \ldots, x_{\pi(k)}, x_{k+2}, \ldots, x_{n} \\
y_{\sigma(1)}, \ldots, y_{\sigma(k-1)}, x_{k+1}, \ldots, x_{n}
\end{array}\right) \mathrm{d} \mu^{\otimes(n-k)}\left(x_{k+1}, \ldots, x_{n}\right) . \\
&=-\left(\begin{array}{c}
n-1 \\
k
\end{array}\right) \frac{1}{k !} \sum_{\sigma \in S_{k}} \operatorname{sgn} \sigma \\
& \quad \int_{Y} \rho\left(x_{k+1}, y_{\sigma(k)}\right)\left(\int_{Y^{n-1-k}} \frac{1}{(n-1) !} \cdot \rho^{\wedge(n-1)}\left(\begin{array}{c}
x_{1}, \ldots, x_{k}, x_{k+2}, \ldots, x_{n} \\
y_{\sigma(1)}, \ldots, y_{\sigma(k-1)}, x_{k+1}, \ldots, x_{n}
\end{array}\right)\right. \\
&\left.\quad \times \mathrm{d} \mu^{\otimes(n-1-k)}\left(x_{k+2}, \ldots, x_{n}\right)\right) \mathrm{d} \mu\left(x_{k+1}\right) .
\end{aligned}
$$

The function $p_{2}: Y^{k} \times Y^{k} \rightarrow \mathbb{K}$, such that $p_{2}\left(x_{1}, \ldots, x_{k}, y_{1}, \ldots, y_{k}\right)$ is $\mu^{\otimes 2 k}$-a.e. equal to expression (3.12), is an integral kernel of the operator

$$
-\left(\begin{array}{c}
n-1 \\
k
\end{array}\right)\left(\mathrm{L}_{n-1}^{k} \rho^{\wedge(n-1)}\right)\left(I^{\otimes(k-1)} \otimes \rho\right) A_{\mathscr{L}^{\prime}}^{(k)}
$$

which occurs on the r.h.s. of (3.1). One concludes that the kernel $\mathcal{L}$ of the operator on the 1.h.s. of (3.1) is $\mu^{\otimes 2 k}$-a.e. equal to the kernel $p_{1}+p_{2}$ of the operator on the r.h.s. of (3.1), which proves the equality of both operators.

The proof of (3.1) for $n=k+1$ and the proof of (3.3) proceed analogously.

Similarly, the proof of (3.2) and (3.4) is accomplished by changing the product $\wedge$ into $\vee$ and replacing determinants in all formulae by pernaments, defined for every complex matrix $A=\left[a_{i, j}\right]_{i, j=1}^{m}$ as

$$
\operatorname{per} A=\sum_{\pi \in S_{m}} a_{\pi(1), 1} \cdots a_{\pi(m), m}
$$

Notice that signs of permutations are omitted in this case, similarly as the multipliers \pm 1 in the Laplace expansions.

Lemma 3.2. Let $k, m \in \mathbb{N}, 1<k<m, \rho \in \mathcal{C}\left(\mathscr{\ell}_{Y}\right), j_{k} \in\{k, \ldots, m\}$, and

$$
R:=\sum_{j_{k-1}=k-1}^{j_{k}-1} \sum_{j_{k-2}=k-2}^{j_{k-1}-1} \cdots \sum_{j_{1}=1}^{j_{2}-1} \rho^{j_{1}} \otimes \rho^{j_{2}-j_{1}} \otimes \cdots \otimes \rho^{j_{k}-j_{k-1}}
$$

(for $k=2$ the only summation index is $\left.j_{k-1}=j_{1}\right)$. Then $A_{\mathscr{d}_{Y}}^{(k)} R=R A_{\mathscr{d}_{Y}}^{(k)}$ and $S_{\mathscr{d}_{Y}}^{(k)} R=R S_{\mathscr{d}_{Y}}^{(k)}$. 
The proof of the above lemma consists in demonstrating the invariance of $R$ under permutations of factors in the tensor products. To this end it suffices to observe that $R$ is invariant under transpositions of neighbouring factors.

Lemma 3.3. Let $\rho \in \tau\left(\mathscr{L}_{Y}\right), \xi_{s}^{\wedge}:=\operatorname{Tr} \rho^{\wedge s}, \xi_{s}^{\vee}:=\operatorname{Tr} \rho^{\vee s}$ for $s \in \mathbb{N}, \xi_{0}^{\wedge}:=1, \xi_{0}^{\vee}:=1$, and

$$
\begin{gathered}
\Pi_{m}^{\wedge p}(\rho):=\sum_{j_{p}=p}^{m} \sum_{j_{p-1}=p-1}^{j_{p}-1} \cdots \sum_{j_{1}=1}^{j_{2}-1} \xi_{m-j_{p}}^{\wedge}(-1)^{p+j_{p}} \rho^{j_{1}} \wedge \rho^{j_{2}-j_{1}} \wedge \cdots \wedge \rho^{j_{p}-j_{p-1}}, \\
\Pi_{m}^{\vee p}(\rho):=\sum_{j_{p}=p}^{m} \sum_{j_{p-1}=p-1}^{j_{p}-1} \cdots \sum_{j_{1}=1}^{j_{2}-1} \xi_{m-j_{p}}^{\vee} \rho^{j_{1}} \vee \rho^{j_{2}-j_{1}} \vee \cdots \vee \rho^{j_{p}-j_{p-1}}
\end{gathered}
$$

for $p, m \in \mathbb{N}, p \leq m$. (For $p=1$ the only summation index is $j_{1}$ and the summation runs over the operators $\rho^{j_{1}}$.) If $2 \leq p<m$ then

$$
\begin{aligned}
& \Pi_{m}^{\wedge p}(\rho)=\left(\Pi_{m-1}^{\wedge(p-1)}(\rho)\right) \wedge \rho-\left(\Pi_{m-1}^{\wedge p}(\rho)\right)\left(I^{\otimes(p-1)} \otimes \rho\right) A_{\mathscr{d l}_{Y},}^{(p)} \\
& \Pi_{m}^{\vee p}(\rho)=\left(\Pi_{m-1}^{\vee(p-1)}(\rho)\right) \vee \rho+\left(\Pi_{m-1}^{\vee p}(\rho)\right)\left(I^{\otimes(p-1)} \otimes \rho\right) S_{\mathscr{L l}_{Y}}^{(p)} .
\end{aligned}
$$

Proof. Equation (3.17) will be first proved for $p>2$. One has

$$
\begin{aligned}
\Pi_{m}^{\wedge p}(\rho)= & \xi_{m-p}^{\wedge} \rho \wedge \cdots \wedge \rho \\
& +\sum_{l_{p}=p}^{m-1} \sum_{l_{p-1}=p-1}^{l_{p}} \sum_{l_{p-2}=p-2}^{l_{p-1}-1} \ldots \sum_{l_{1}=1}^{l_{2}-1} \xi_{m-l_{p}-1}^{\wedge}(-1)^{p+l_{p}+1} \rho^{l_{1}} \wedge \rho^{l_{2}-l_{1}} \wedge \cdots \wedge \rho^{l_{p-1}-l_{p-2}} \wedge \rho^{l_{p}-l_{p-1}+1} \\
= & \xi_{m-p}^{\wedge} \rho \wedge \cdots \wedge \rho \\
& +\sum_{l_{p}=p}^{m-1} \sum_{l_{p-1}=p-1}^{l_{p}-1} \sum_{l_{p-2}=p-2}^{l_{p-1}-1} \cdots \sum_{l_{1}=1}^{l_{2}-1} \xi_{m-l_{p}-1}^{\wedge}(-1)^{p+l_{p}+1} \rho^{l_{1}} \wedge \rho^{l_{2}-l_{1}} \wedge \cdots \wedge \rho^{l_{p-1}-l_{p-2}} \wedge \rho^{l_{p}-l_{p-1}+1} \\
& +\sum_{l_{p}=p}^{m-1} \sum_{l_{p-2}=p-2}^{l_{p}-1} \sum_{l_{p-3}=p-3}^{l_{p-2}-1} \ldots \sum_{l_{1}=1}^{l_{2}-1} \xi_{m-l_{p}-1}^{\wedge}(-1)^{p+l_{p}+1} \rho^{l_{1}} \wedge \rho^{l_{2}-l_{1}} \wedge \cdots \wedge \rho^{l_{p-2}-l_{p-3}} \wedge \rho^{l_{p}-l_{p-2}} \wedge \rho .
\end{aligned}
$$

The first and the third term after the last of equalities (3.20) yield

$$
\sum_{j_{p-1}=p-1}^{m-1} \sum_{j_{p-2}=p-2}^{j_{p-1}-1} \cdots \sum_{j_{1}=1}^{j_{2}-1}\left(\xi_{(m-1)-j_{p-1}}^{\wedge}(-1)^{(p-1)+j_{p-1}} \rho^{j_{1}} \wedge \rho^{j_{2}-j_{1}} \wedge \cdots \wedge \rho^{j_{p-1}-j_{p-2}}\right) \wedge \rho
$$


for $l_{p} \equiv j_{p-1}, l_{p-2} \equiv j_{p-2}, \ldots, l_{1} \equiv j_{1}$. By Lemma 3.2, the second term after the last of equalities (3.20) equals

$$
-\left(\Pi_{m-1}^{\wedge p}(\rho)\right)\left(I^{\otimes(p-1)} \otimes \rho\right) A_{\mathscr{d l}_{Y}}^{(p)}
$$

The sum of expressions (3.21) and (3.22) is equal to the r.h.s. of (3.17) for $p>2$. After simplifications the proof also applies to the case of $p=2$.

The proof of (3.18) is analogous to that of (3.17).

The next theorem provides the explicit form of $(n, k)$-contractions of product operators. The proof for $k=1,2$ was given in [11, 12]. The author of [12] emphasized that formula (3.23) for $k=2$ was derived by S. Pruski in 1978.

Theorem 3.4 (Explicit formulae). Let $k, n \in \mathbb{N}, k<n, \rho \in \mathcal{C}\left(\mathcal{L}_{Y}\right), \xi_{s}^{\wedge}:=\operatorname{Tr} \rho^{\wedge s}, \xi_{s}^{\vee}:=\operatorname{Tr} \rho^{\vee s}$ for $s \in \mathbb{N}$, and $\xi_{0}^{\wedge}:=1, \xi_{0}^{\vee}:=1$. Then

$$
\begin{aligned}
\left(\begin{array}{l}
n \\
k
\end{array}\right) \mathrm{L}_{n}^{k} \rho^{\wedge n} & =\sum_{j_{k}=k}^{n} \sum_{j_{k-1}=k-1}^{j_{k}-1} \cdots \sum_{j_{1}=1}^{j_{2}-1} \xi_{n-j_{k}}^{\wedge}(-1)^{k+j_{k}} \rho^{j_{1}} \wedge \rho^{j_{2}-j_{1}} \wedge \cdots \wedge \rho^{j_{k}-j_{k-1}} \\
& =\sum_{i_{1}=1}^{n-(k-1)} \sum_{i_{2}=1}^{n-i_{1}-(k-2)} \cdots \sum_{i_{k-1}=1}^{n-i_{1}-\cdots-i_{k-2}-1} \sum_{i_{k}=1}^{n-i_{1}-\cdots-i_{k-1}} \xi_{n-i_{1}-\cdots-i_{k}}^{\wedge}(-1)^{k+i_{1}+\cdots+i_{k}} \rho^{i_{1}} \wedge \cdots \wedge \rho^{i_{k}}, \\
\left(\begin{array}{l}
n \\
k
\end{array}\right) \mathrm{L}_{n}^{k} \rho^{\vee n} & =\sum_{j_{k}=k}^{n} \sum_{j_{k-1}=k-1}^{j_{k}-1} \cdots \sum_{j_{1}=1}^{j_{2}-1} \xi_{n-j_{k}}^{\vee} \rho^{j_{1}} \vee \rho^{j_{2}-j_{1}} \vee \cdots \vee \rho^{j_{k}-j_{k-1}} \\
& =\sum_{i_{1}=1}^{n-(k-1)} \sum_{i_{2}=1}^{n-i_{1}-(k-2)} \cdots \sum_{i_{k-1}=1}^{n-i_{1}-\cdots-i_{k-2}-1} \sum_{i_{k}=1}^{n-i_{1}-\cdots-i_{k-1}} \xi_{n-i_{1}-\cdots-i_{k}}^{\vee} \rho^{i_{1}} \vee \cdots \vee \rho^{i_{k}} .
\end{aligned}
$$

(For $k=1$ the only summation indices are $j_{1}$ and $i_{1}$ and the summation runs over the operators $\rho^{j_{1}}$ and $\rho^{i_{1}}$, resp.)

Proof. For every $p, m \in \mathbb{N}, p \leq m$, let $\Pi_{m}^{\wedge p}(\rho)$ be defined as in Lemma 3.3. Then the first of equalities (3.23) can be written as

$$
\left(\begin{array}{l}
n \\
k
\end{array}\right) \mathrm{L}_{n}^{k} \rho^{\wedge n}=\Pi_{n}^{\wedge k}(\rho)
$$

The proof of (3.25) will be carried out by (double) induction with respect to $k$ and, for fixed $k$, with respect to $n>k$.

$\left(1^{\circ}\right)(k=1)$ This part of the proof is by induction with respect to $n>1$.

(a) $(n=2)$ According to Theorem 3.1, $2 \mathrm{~L}_{2}^{1} \rho^{\wedge 2}=(\operatorname{Tr} \rho) \rho-\rho^{2}=\Pi_{2}^{\wedge 1}(\rho)$.

(b) Assuming validity of formula (3.25) (with $k=1$ ) for $n \in\{2, \ldots, m-1\}$, where $m \in \mathbb{N}, m>2$, its validity will be shown for $n=m$. 
One has

$$
\Pi_{m}^{\wedge 1}(\rho)=\xi_{m-1}^{\wedge} \rho+\sum_{j_{1}=2}^{m} \xi_{m-j_{1}}^{\wedge}(-1)^{1+j_{1}} \rho^{j_{1}}=\xi_{m-1}^{\wedge} \rho-\left(\Pi_{m-1}^{\wedge 1}(\rho)\right) \rho .
$$

Thus, according to the inductive hypothesis for $n \in\{2, \ldots, m-1\}$,

$$
\Pi_{m}^{\wedge 1}(\rho)=\xi_{m-1}^{\wedge} \rho-(m-1)\left(\mathrm{L}_{m-1}^{1} \rho^{\wedge(m-1)}\right) \rho,
$$

which, in view of Theorem 3.1, yields $\left(\begin{array}{c}m \\ 1\end{array}\right) \mathrm{L}_{m}^{1} \rho^{\wedge m}=\Pi_{m}^{\wedge 1}(\rho)$.

$\left(2^{\circ}\right)$ Assuming validity of formula (3.25) for $k \in\{1, \ldots, p-1\}$ (and every $n>k$ ), where $p \in \mathbb{N}, p>1$, its validity will be shown for $k=p$. For arbitrarily fixed $p$ the proof will be carried out by induction with respect to $n>p$.

(a) $(n=p+1)$ By the inductive hypothesis with respect to $k$ and Lemma 3.3,

$$
\Pi_{p+1}^{\wedge p}(\rho)=\left(\begin{array}{c}
(p+1)-1 \\
p-1
\end{array}\right)\left(\mathrm{L}_{(p+1)-1}^{p-1} \rho^{\wedge((p+1)-1)}\right) \wedge \rho-\rho^{\wedge p}\left(I^{\otimes(p-1)} \otimes \rho\right) A_{\mathscr{d}^{\prime}}^{(p)}
$$

hence $\left(\begin{array}{c}p+1 \\ p\end{array}\right) \mathrm{L}_{p+1}^{p} \rho^{\wedge(p+1)}=\Pi_{p+1}^{\wedge p}(\rho)$, according to Theorem 3.1.

(b) Assuming validity of formula (3.25) for $n \in\{p+1, \ldots, m-1\}$, where $k=p$, $m \in \mathbb{N}, m>p+1$, its validity will be shown for $n=m$.

By the inductive hypothesis for $k \in\{1, \ldots, p-1\}$ and Lemma 3.3 one has

$$
\Pi_{m}^{\wedge p}(\rho)=\left(\begin{array}{c}
m-1 \\
p-1
\end{array}\right)\left(\mathrm{L}_{m-1}^{p-1} \rho^{\wedge(m-1)}\right) \wedge \rho-\left(\Pi_{m-1}^{\wedge p}(\rho)\right)\left(I^{\otimes(p-1)} \otimes \rho\right) A_{\mathscr{L}_{Y}}^{(p)}
$$

According to the inductive hypothesis for $n \in\{p+1, \ldots, m-1\}$ one thus obtains

$$
\Pi_{m}^{\wedge p}(\rho)=\left(\begin{array}{c}
m-1 \\
p-1
\end{array}\right)\left(\mathrm{L}_{m-1}^{p-1} \rho^{\wedge(m-1)}\right) \wedge \rho-\left(\begin{array}{c}
m-1 \\
p
\end{array}\right)\left(\mathrm{L}_{m-1}^{p} \rho^{\wedge(m-1)}\right)\left(I^{\otimes(p-1)} \otimes \rho\right) A_{\mathscr{d}^{\prime}}^{(p)}
$$

which, in view of Theorem 3.1, yields $\left(\begin{array}{c}m \\ p\end{array}\right) \mathrm{L}_{m}^{p} \rho^{\wedge m}=\Pi_{m}^{\wedge p}(\rho)$. This completes the inductive proof for (3.25) with respect to $n>p$ and with respect to $k$.

Now turn to the second of equalities (3.23). For $k=1$ it is identity. Let $k \geq 2$. Setting $j_{1}=i_{1}, j_{2}=i_{1}+i_{2}, \ldots, j_{k}=i_{1}+\cdots+i_{k}$ or, equivalently, $i_{1}=j_{1}, i_{2}=j_{2}-j_{1}, i_{3}=j_{3}-j_{2}, \ldots$, $i_{k}=j_{k}-j_{k-1}$, one checks that both sides of the equality in question are equal to

$$
\sum_{j_{1}=1}^{n-(k-1)} \sum_{j_{2}=j_{1}+1}^{n-(k-2)} \cdots \sum_{j_{k-1}=j_{k-2}+1}^{n-1} \sum_{j_{k}=j_{k-1}+1}^{n} \xi_{n-j_{k}}^{\wedge}(-1)^{k+j_{k}} \rho^{j_{1}} \wedge \rho^{j_{2}-j_{1}} \wedge \cdots \wedge \rho^{j_{k}-j_{k-1}}
$$

The proof of (3.24) is analogous to that of (3.23). 


\section{Asymptotic Form for Contractions of Product States}

The explicit forms of the contractions of product states given by Theorem 3.4 are quite complex. In the present section they are replaced by simpler operators, equivalent in the thermodynamic limit. The main results in this section are Theorems 4.9 and 4.14.

In what follows use is made of the Hilbert space $\mathscr{H}_{\Omega}:=L^{2}(\Omega, \mu)$ (over $\mathbb{C}$ or $\mathbb{R}$ ), where the measure $\mu$ is separable, $\sigma$-finite, and satisfies the condition $\mu(\Omega)=+\infty$. For every $\mu$ measurable subset $Y \subset \Omega$ it is assumed $\mathscr{L}_{Y}:=L^{2}(Y, \mu)$.

Let $\mathcal{M}(\Omega)$ be a fixed family of measurable subsets of $\Omega$ such that $0<\mu(Y)<+\infty$ for every $Y \in \mathcal{M}(\Omega)$ (it can be the family of all such subsets). Fix $d \in \mathbb{R}, d>0$, and assume that there exists a sequence $\left\{Y_{n}\right\}_{n \in \mathbb{N}} \subset \mathcal{M}(\Omega)$ such that $n / \mu\left(Y_{n}\right) \rightarrow d$ as $n \rightarrow \infty$.

Definition 4.1. Fix $d \in \mathbb{R}, d>0$, and let $\left\{b_{Y, n}\right\}_{(Y, n) \in \mathcal{M}(\Omega) \times \mathbb{N}}$ be a family of complex numbers. A complex number $b$ is said to be the thermodynamic limit of this family if for every sequence $\left\{Y_{n}\right\}_{n \in \mathbb{N}} \subset \mathcal{M}(\Omega)$ such that $\lim _{n \rightarrow \infty} n / \mu\left(Y_{n}\right)=d$ the condition $\lim _{n \rightarrow \infty} b_{Y_{n}, n}=b$ is fulfilled. In such a case $b$ is denoted by $d-\lim _{n, \mu(Y) \rightarrow \infty} b_{Y, n}$.

Special attention will be given to the families of complex numbers of the form $\operatorname{Tr}\left(\mathrm{L}_{n}^{k} K_{Y, n}\right) C_{Y}$, where $k, n \in \mathbb{N}, n>k, K_{Y, n} \in \tau\left(\mathscr{\ell}_{Y}^{\otimes n}\right)$, and $C_{Y} \in B\left(\mathcal{H}_{Y}^{\otimes k}\right)$.

Definition 4.1 does not guarantee the convergence of families $\left\{b_{Y, n}\right\}$ of interest in physics. To obtain such a convergence, additional conditions (such as conditions of uniform growth [18]) are usually imposed on the sequence $\left\{Y_{n}\right\}_{n \in \mathbb{N}}$ in question. However, those additional conditions do not affect considerations in this paper.

Expression of expectation values of observables in mixed states by using trace, mentioned in Introduction, is the motivation for the following definition.

Definition 4.2. Fix $k \in \mathbb{N}$ and $d \in \mathbb{R}, d>0$. Families $\left\{A_{Y, n}\right\}_{(Y, n) \in \mathcal{M}(\Omega) \times \mathbb{N}}$ and $\left\{B_{Y, n}\right\}_{(Y, n) \in \mathcal{M}(\Omega) \times \mathbb{N}}$ of operators $A_{Y, n}, B_{Y, n} \in \tau\left(\mathscr{L}_{Y}^{\otimes k}\right)$ are said to be asymptotically equivalent (symbolically: $\left.A_{Y, n} \approx B_{Y, n}\right)$, if for every family $\left\{C_{Y, n}\right\}_{(Y, n) \in \mathcal{M}(\Omega) \times \mathbb{N}}$ of operators $C_{Y, n} \in \mathcal{B}\left(\mathcal{L}_{Y}^{\otimes k}\right)$ with uniformly bounded operator norms one has

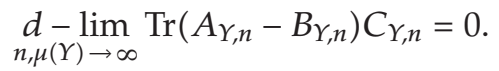

Condition (4.1) is required to hold in particular for families $\left\{C_{Y, n}\right\}_{(Y, n) \in \mathcal{M}(\Omega) \times \mathbb{N}}$ such that $C_{Y, n}=C_{Y, m}$ for all $Y \in \mathcal{M}(\Omega), n, m \in \mathbb{N}$.

Remark 4.3. The authors of $[11,12]$ used some different definition of asymptotic equivalence of families of operators, closer to Definition 4.10 in this paper.

Remark 4.4. For fixed $k \in \mathbb{N}$ and $d \in \mathbb{R}, d>0$, the relation $\approx$ is an equivalence relation. If $A_{Y, n} \approx B_{Y, n}$ then for every family of operators $C_{Y_{, n}}$ as in Definition 4.2 the limit

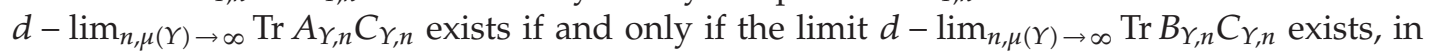
which case both limits are equal. Notice also that if $A_{Y, n} \approx B_{Y, n}$ then $A_{Y, n}+D_{Y, n} \approx B_{Y, n}+D_{Y, n}$ and $a A_{Y, n} \approx a B_{Y, n}$ for every family $\left\{D_{Y, n}\right\}_{(Y, n) \in \mathcal{M}(\Omega) \times \mathbb{N}} \subset \tau\left(\mathcal{L}_{Y}^{\otimes k}\right)$ and $a \in \mathbb{C}$. Furthermore, for every family $\left\{A_{Y, n}\right\}_{(Y, n) \in \mathcal{M}(\Omega) \times \mathbb{N}} \subset \mathcal{C}\left(\mathscr{L}_{Y}^{\otimes k}\right)$ with uniformly bounded trace norms $\operatorname{Tr}\left|A_{Y, n}\right|$ and for every sequence $\left\{a_{n}\right\}_{n \in \mathbb{N}} \subset \mathbb{C}$ convergent to $a \in \mathbb{C}$ one has $a_{n} A_{Y, n} \approx a A_{Y, n}$. 
International Journal of Mathematics and Mathematical Sciences

Lemma 4.5. Let $\left\{A_{Y, n}\right\}_{(Y, n) \in \mathcal{M}(\Omega) \times \mathbb{N}}$ and $\left\{B_{Y, n}\right\}_{(Y, n) \in \mathcal{M}(\Omega) \times \mathbb{N}}$ be as in Definition 4.2. Then

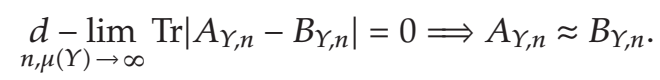

Moreover, if the operators $A_{Y, n}, B_{Y, n}$ are self-adjoint then

$$
A_{Y, n} \approx B_{Y, n} \Longrightarrow \underset{n, \mu(Y) \rightarrow \infty}{d-\lim _{1}} \operatorname{Tr}\left|A_{Y, n}-B_{Y, n}\right|=0
$$

Proof. Implication (4.2) follows from Definition 4.2 and the estimate

$$
\left|\operatorname{Tr}\left(A_{Y, n}-B_{Y, n}\right) C_{Y, n}\right| \leq\left\|C_{Y, n}\right\| \operatorname{Tr}\left|A_{Y, n}-B_{Y, n}\right| .
$$

Now assume that $A_{Y, n} \approx B_{Y, n}$, which is equivalent to the condition

$$
D_{Y, n} \approx 0,
$$

where $D_{Y, n}:=A_{Y, n}-B_{Y, n}$. The operators $D_{Y, n}$ have the spectral representations

$$
D_{Y, n}=\sum_{i=1}^{\infty} \lambda_{i}(Y, n) P_{\varphi_{i}(Y, n)}
$$

where $P_{\varphi_{i}(Y, n)}$ are the projectors onto orthogonal one dimensional subspaces of eigenvectors $\varphi_{i}(Y, n)$ of $D_{Y, n}$, corresponding to eigenvalues $\lambda_{i}(Y, n) \in \mathbb{R}$. Since $\sum_{i=1}^{\infty}\left|\lambda_{i}(Y, n)\right|=\operatorname{Tr}\left|D_{Y, n}\right|<$ $+\infty$, for every $(Y, n) \in \mathcal{M}(\Omega) \times \mathbb{N}$ there exists $m(Y, n) \in \mathbb{N}$ such that $\sum_{i=m(Y, n)+1}^{\infty}\left|\lambda_{i}(Y, n)\right|<1 / n$. Thus the operators

$$
F_{Y, n}=\sum_{i=1}^{m(Y, n)} \lambda_{i}(Y, n) P_{\varphi_{i}(Y, n)}
$$

satisfy the condition

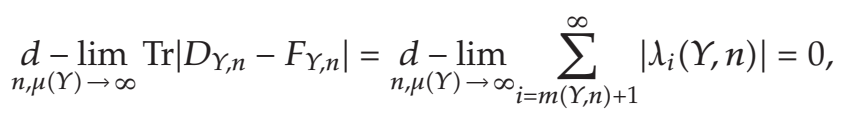

which, in view of implication (4.2) proved and condition (4.5), yields $F_{Y, n} \approx D_{Y, n} \approx 0$. In particular,

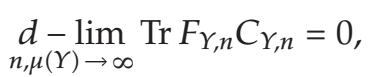


where

$$
C_{Y, n}=\sum_{i=1}^{m(Y, n)} \operatorname{sgn}\left(\lambda_{i}(Y, n)\right) P_{\varphi_{i}(Y, n)}, \quad\left\|C_{Y, n}\right\|=1 .
$$

Observe that $\operatorname{Tr} F_{Y, n} C_{Y, n}=\operatorname{Tr}\left|F_{Y, n}\right|$, hence condition (4.9) gives

$$
\underset{n, \mu(Y) \rightarrow \infty}{d-\lim _{n}} \operatorname{Tr}\left|F_{Y, n}\right|=0
$$

Since $\operatorname{Tr}\left|D_{Y, n}\right| \leq \operatorname{Tr}\left|D_{Y, n}-F_{Y, n}\right|+\operatorname{Tr}\left|F_{Y, n}\right|$, conditions (4.8) and (4.11) yield

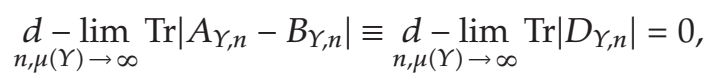

which proves implication (4.3).

The following lemma follows from Lemma 4.5.

Lemma 4.6. Fix $k, m \in \mathbb{N}$. Let $\left\{A_{Y, n}\right\}_{(Y, n) \in \mathcal{M}(\Omega) \times \mathbb{N}}$ and $\left\{B_{Y, n}\right\}_{(Y, n) \in \mathcal{M}(\Omega) \times \mathbb{N}}$ be families of self-adjoint operators $A_{Y, n}, B_{Y, n} \in \mathcal{\tau}\left(\mathscr{L}_{Y}^{\otimes k}\right)$ such that $A_{Y, n} \approx B_{Y, n}$, and let $\left\{D_{Y, n}\right\}_{(Y, n) \in \mathcal{M}(\Omega) \times \mathbb{N}}$ be a family of operators $D_{Y, n} \in \tau\left(\mathscr{\ell}_{Y}^{\otimes m}\right)$ with uniformly bounded trace norms $\operatorname{Tr}\left|D_{Y, n}\right|$. Then

$$
\begin{aligned}
A_{Y, n} \otimes D_{Y, n} \approx B_{Y, n} \otimes D_{Y, n}, & & D_{Y, n} \otimes A_{Y, n} \approx D_{Y, n} \otimes B_{Y, n}, \\
A_{Y, n} \wedge D_{Y, n} \approx B_{Y, n} \wedge D_{Y, n,} & & D_{Y, n} \wedge A_{Y, n} \approx D_{Y, n} \wedge B_{Y, n,} \\
A_{Y, n} \vee D_{Y, n} \approx B_{Y, n} \vee D_{Y, n,} & & D_{Y, n} \vee A_{Y, n} \approx D_{Y, n} \vee B_{Y, n} .
\end{aligned}
$$

In the sequel $\left\{\rho_{Y}\right\}_{Y \in \mathcal{M}(\Omega)}$ denotes a family of nonnegative definite self-adjoint operators $\rho_{Y} \in \tau\left(\mathscr{\ell}_{Y}\right)$, and for every $(Y, n) \in \mathcal{M}(\Omega) \times \mathbb{N}$ it is assumed that

$$
\begin{gathered}
\xi_{Y, 0}^{\wedge}:=1, \quad \xi_{Y, 0}^{\vee}:=1, \quad \rho_{Y}^{\wedge 1}:=\rho_{Y}, \quad \rho_{Y}^{\vee 1}:=\rho_{Y}, \\
\xi_{Y, n}^{\wedge}:=\operatorname{Tr} \rho_{Y}^{\wedge n}>0, \quad \xi_{Y, n}^{\vee}:=\operatorname{Tr} \rho_{Y}^{\vee n}>0, \\
s_{Y, n}^{\wedge}:=\frac{\xi_{Y, n-1}^{\wedge}}{\xi_{Y, n}^{\wedge}}, \quad s_{Y, n}^{\vee}:=\frac{\xi_{Y, n-1}^{\vee}}{\xi_{Y, n}^{\vee}} .
\end{gathered}
$$

The objective of this section is to find density operators of the most simple form which are asymptotically equivalent to the operators

$$
\hat{\sigma}_{Y, n}^{(k)}:=\mathrm{L}_{n}^{k}\left(\frac{1}{\xi_{Y, n}^{\wedge}} \rho_{Y}^{\wedge n}\right), \quad \stackrel{{ }^{\vee}}{(k)}:=\mathrm{L}_{n}^{k}\left(\frac{1}{\xi_{Y, n}^{\vee}} \rho_{Y}^{\vee n}\right),
$$

defined for fixed $k \in \mathbb{N}$ and every $(Y, n) \in \mathcal{M}(\Omega) \times \mathbb{N}, n>k$. 
Remark 4.7. For every $(Y, n) \in \mathcal{M}(\Omega) \times \mathbb{N}$ the operator $I+s_{Y, n+1}^{\wedge} \rho_{Y}$ is invertible and $\left\|\left(I+s_{Y, n+1}^{\wedge} \rho_{Y}\right)^{-1}\right\|=1$. Furthermore, if $s_{Y, n+1}^{\vee}\left\|\rho_{Y}\right\|<1$ then $I-s_{Y, n+1}^{\vee} \rho_{Y}$ is invertible and $\left\|\left(I-s_{Y, n+1}^{\vee} \rho_{Y}\right)^{-1}\right\|=\left(1-s_{Y, n+1}^{\vee}\left\|\rho_{Y}\right\|\right)^{-1}$.

The next theorem is a version of a theorem studied in [11, 12] (see Remark 4.3).

Theorem 4.8. If $\hat{\sigma}_{Y, n}^{(1)} \approx \hat{\sigma}_{Y, n+1}^{(1)}$ and the reals $s_{Y, n+1}^{\wedge}\left\|\rho_{Y}\right\|,(Y, n) \in \mathcal{M}(\Omega) \times \mathbb{N}$, are uniformly bounded then

$$
\begin{aligned}
& \hat{\sigma}_{Y, n}^{(1)}\left(I+s_{Y, n+1}^{\wedge} \rho_{Y}\right) \approx(n+1)^{-1} s_{Y, n+1}^{\wedge} \rho_{Y}, \\
& \hat{\sigma}_{Y, n}^{(1)} \approx(n+1)^{-1} s_{Y, n+1}^{\wedge} \rho_{Y}\left(I+s_{Y, n+1}^{\wedge} \rho_{Y}\right)^{-1} .
\end{aligned}
$$

If $\stackrel{V^{(1)}}{\sigma_{Y, n}} \approx \stackrel{\vee}{\sigma}_{Y, n+1}^{(1)}$ and the reals $s_{Y, n}^{\vee}\left\|\rho_{Y}\right\|,(Y, n) \in \mathcal{M}(\Omega) \times \mathbb{N}$, are uniformly bounded then

$$
\stackrel{{ }^{\vee}(1)}{\sigma_{Y, n}}\left(I-s_{Y, n+1}^{\vee} \rho_{Y}\right) \approx(n+1)^{-1} s_{Y, n+1}^{\vee} \rho_{Y}
$$

If, additionally, $s_{Y, n}^{\vee}\left\|\rho_{Y}\right\| \leq \epsilon$ for some $\epsilon<1$ and every $(Y, n) \in \mathcal{M}(\Omega) \times \mathbb{N}$ then

$$
\stackrel{\vee^{(1)}}{\sigma_{Y, n}} \approx(n+1)^{-1} s_{Y, n+1}^{\vee} \rho_{Y}\left(I-s_{Y, n+1}^{\vee} \rho_{Y}\right)^{-1}
$$

Proof. By Theorem 3.1 and the assumption $\hat{\sigma}_{Y, n}^{(1)} \approx \hat{\sigma}_{Y, n+1}^{(1)}$ one has

$$
\hat{\sigma}_{Y, n}^{(1)}-(n+1)^{-1} s_{Y, n+1}^{\wedge} \rho_{Y} \approx-(n+1)^{-1} n \hat{\sigma}_{Y, n}^{(1)}\left(s_{Y, n+1}^{\wedge} \rho_{Y}\right) .
$$

Since $\operatorname{Tr}\left|\hat{\sigma}_{Y, n}^{(1)}\left(s_{Y, n+1}^{\wedge} \rho_{Y}\right)\right| \leq s_{Y, n+1}^{\wedge}\left\|\rho_{Y}\right\| \operatorname{Tr}\left|\hat{\sigma}_{Y, n}^{(1)}\right|=s_{Y, n+1}^{\wedge}\left\|\rho_{Y}\right\|$, relation (4.20) yields (4.16), in view of Remark 4.4.

Now turn to the proof of relation (4.17). According to Remark 4.7,

$$
\begin{aligned}
\operatorname{Tr}\left|\hat{\sigma}_{Y, n}^{(1)}-(n+1)^{-1} s_{Y, n+1}^{\wedge} \rho_{Y}\left(I+s_{Y, n+1}^{\wedge} \rho_{Y}\right)^{-1}\right| \\
\quad \leq\left\|\left(I+s_{Y, n+1}^{\wedge} \rho_{Y}\right)^{-1}\right\| \operatorname{Tr}\left|\hat{\sigma}_{Y, n}^{(1)}\left(I+s_{Y, n+1}^{\wedge} \rho_{Y}\right)-(n+1)^{-1} s_{Y, n+1}^{\wedge} \rho_{Y}\right| \\
\quad=\operatorname{Tr}\left|\hat{\sigma}_{Y, n}^{(1)}\left(I+s_{Y, n+1}^{\wedge} \rho_{Y}\right)-(n+1)^{-1} s_{Y, n+1}^{\wedge} \rho_{Y}\right| .
\end{aligned}
$$

The explicit form of $\hat{\sigma}_{Y, n}^{(1)}$ given by Theorem 3.4 shows that $\hat{\sigma}_{Y, n}^{(1)}$ commutes with $I+s_{Y, n+1}^{\wedge} \rho_{Y}$, and since both operators are self-adjoint, $\hat{\sigma}_{Y, n}^{(1)}\left(I+s_{Y, n+1}^{\wedge} \rho_{Y}\right)$ is also self-adjoint. Thus conditions (4.16), (4.21), and Lemma 4.5 yield (4.17). 
The proof of relations (4.18), (4.19) runs parallel to that of (4.16), (4.17). Notice that in this case the expression $\left\|\left(I+s_{Y, n+1}^{\wedge} \rho_{Y}\right)^{-1}\right\|=1$ from estimate (4.21) is replaced by $\left\|\left(I-s_{Y, n+1}^{\wedge} \rho_{Y}\right)^{-1}\right\|=\left(1-s_{Y, n+1}^{\wedge}\left\|\rho_{Y}\right\|\right)^{-1} \leq(1-\epsilon)^{-1}$ (see Remark 4.7).

The following theorem for $k=2$ (with the reservation of Remark 4.3) was obtained in $[11,12]$. The author of [12] gave also arguments that can be used to check the assumptions of this theorem.

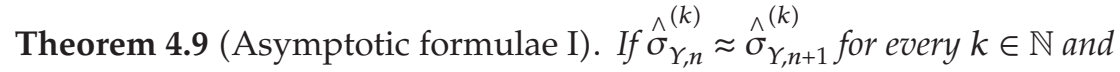

$$
s_{Y, n}^{\wedge}\left\|\rho_{Y}\right\| \leq 2 \text { for every }(Y, n) \in \mathcal{M}(\Omega) \times \mathbb{N}
$$

then, for every $k \in \mathbb{N}, k \geq 2$,

$$
\hat{\sigma}_{Y, n}^{(k)} \approx k ! \underbrace{\hat{\sigma}_{Y, n}^{(1)} \wedge \cdots \wedge \hat{\sigma}_{Y, n}^{(1)}}_{k} .
$$

If $\stackrel{\vee}{\sigma}_{Y, n}^{(k)} \approx \stackrel{\vee}{\sigma}^{(k)}$ for every $k \in \mathbb{N}$ and

$$
s_{Y, n}^{\vee}\left\|\rho_{Y}\right\| \leq \epsilon \text { for some } \epsilon<1 \text { and every }(Y, n) \in \mathcal{M}(\Omega) \times \mathbb{N}
$$

then, for every $k \in \mathbb{N}, k \geq 2$,

$$
\stackrel{\vee^{(k)}}{\sigma_{Y, n}} \approx k ! \underbrace{\stackrel{\vee^{(1)}}{\sigma_{Y, n} \vee \cdots \vee} \stackrel{\vee}{\sigma}_{Y, n}^{(1)}}_{k} .
$$

Proof. First equivalence (4.23) will be proved. Observe that

$$
\begin{aligned}
& 2 \operatorname{Tr}\left|\hat{\sigma}_{Y, n}^{(q)}-q ! \hat{\sigma}_{Y, n}^{(1)} \wedge \cdots \wedge \hat{\sigma}_{Y, n}^{(1)}\right| \\
&=\operatorname{Tr} \mid\left(\hat{\sigma}_{Y, n}^{(q)}-q ! \hat{\sigma}_{Y, n}^{(1)} \wedge \cdots \wedge \hat{\sigma}_{Y, n}^{(1)}\right)\left(I^{\otimes(q-1)} \otimes\left(I+s_{Y, n+1}^{\wedge} \rho_{Y}\right)\right) A_{\mathscr{L}_{Y}}^{(q)} \\
& \quad+\left(\hat{\sigma}_{Y, n}^{(q)}-q ! \hat{\sigma}_{Y, n}^{(1)} \wedge \cdots \wedge \hat{\sigma}_{Y, n}^{(1)}\right)\left(I^{\otimes(q-1)} \otimes\left(I-s_{Y, n+1}^{\wedge} \rho_{Y}\right)\right) A_{\mathscr{L}_{Y}}^{(q)} \mid \\
& \leq \operatorname{Tr}\left|\left(\hat{\sigma}_{Y, n}^{(q)}-q ! \hat{\sigma}_{Y, n}^{(1)} \wedge \cdots \wedge \hat{\sigma}_{Y, n}^{(1)}\right)\left(I^{\otimes(q-1)} \otimes\left(I+s_{Y, n+1}^{\wedge} \rho_{Y}\right)\right) A_{\mathscr{L}_{Y}}^{(q)}\right| \\
& \quad+\left\|I-s_{Y, n+1}^{\wedge} \rho_{Y}\right\| \operatorname{Tr}\left|\hat{\sigma}_{Y, n}^{(q)}-q ! \hat{\sigma}_{Y, n}^{(1)} \wedge \cdots \wedge \hat{\sigma}_{Y, n}^{(1)}\right|
\end{aligned}
$$


hence

$$
\begin{aligned}
(2- & \left.\left\|I-s_{Y, n+1}^{\wedge} \rho_{Y}\right\|\right) \operatorname{Tr}\left|\hat{\sigma}_{Y, n}^{(q)}-q ! \hat{\sigma}_{Y, n}^{(1)} \wedge \cdots \wedge \hat{\sigma}_{Y, n}^{(1)}\right| \\
& \leq \operatorname{Tr}\left|\left(\hat{\sigma}_{Y, n}^{(q)}-q ! \hat{\sigma}_{Y, n}^{(1)} \wedge \cdots \wedge \hat{\sigma}_{Y, n}^{(1)}\right)\left(I^{\otimes(q-1)} \otimes\left(I+s_{Y, n+1}^{\wedge} \rho_{Y}\right)\right) A_{\mathscr{d}_{Y}}^{(q)}\right| .
\end{aligned}
$$

Since the operators $\rho_{Y}$ are trace class, $\inf _{\varphi \in \mathscr{C}_{Y} ;\|\varphi\|=1}\left\langle\varphi, \rho_{Y} \varphi\right\rangle=0$. Thus, by assumption (4.22) and the self-adjointness of the operators $I-s_{Y, n+1}^{\wedge} \rho_{Y}$, one obtains

$$
\begin{aligned}
\left\|I-s_{Y, n+1}^{\wedge} \rho_{Y}\right\| & =\sup _{\substack{\varphi \in \mathscr{L}_{Y} \\
\|\varphi\|=1}}\left|\left\langle\varphi,\left(I-s_{Y, n+1}^{\wedge} \rho_{Y}\right) \varphi\right\rangle\right| \\
& =\max \left\{\begin{array}{c}
1-s_{Y, n+1}^{\wedge} \inf _{\substack{\varphi \in \mathscr{C}_{Y} \\
\|\varphi\|=1}}\left\langle\varphi, \rho_{Y} \varphi\right\rangle, s_{Y, n+1}^{\wedge} \sup _{\substack{\varphi \in \mathscr{L}_{Y} \\
\|\varphi\|=1}}\left\langle\varphi, \rho_{Y} \varphi\right\rangle-1 \\
\|=1 .
\end{array}\right.
\end{aligned}
$$

The rest of the proof of (4.23) is by induction with respect to $k \geq 2$.

$\left(1^{\circ}\right)(k=2)$ By Theorem 3.1 for $n \geq 2$ one has

$$
\begin{aligned}
\frac{1}{(n+1)^{2}}\left(\begin{array}{c}
n+1 \\
2
\end{array}\right) \hat{\sigma}_{Y, n+1}^{(2)}= & \frac{n}{n+1} \hat{\sigma}_{Y, n}^{(1)} \wedge\left((n+1)^{-1} s_{Y, n+1}^{\wedge} \rho_{Y}\right) \\
& -\frac{1}{(n+1)^{2}}\left(\begin{array}{l}
n \\
2
\end{array}\right) \hat{\sigma}_{Y, n}^{(2)}\left(I \otimes\left(s_{Y, n+1}^{\wedge} \rho_{Y}\right)\right) A_{\mathscr{d Y}}^{(2)} .
\end{aligned}
$$

Assumption (4.22) gives $\operatorname{Tr}\left|\hat{\sigma}_{Y, n}^{(2)}\left(I \otimes\left(s_{Y, n+1}^{\wedge} \rho_{Y}\right)\right) A_{\mathscr{d}_{Y}}^{(2)}\right| \leq s_{Y, n+1}^{\wedge}\left\|\rho_{Y}\right\| \operatorname{Tr}\left|\hat{\sigma}_{Y, n}^{(2)}\right| \leq 2$, hence, by (4.29), Remark 4.4, and the assumption $\hat{\sigma}_{Y, n}^{(2)} \approx \hat{\sigma}_{Y, n+1}^{(2)}$, one obtains

$$
\hat{\sigma}_{Y, n}^{(2)}+\hat{\sigma}_{Y, n}^{(2)}\left(I \otimes\left(s_{Y, n+1}^{\wedge} \rho_{Y}\right)\right) A_{\mathscr{d}_{Y}}^{(2)} \approx 2 \frac{n}{n+1} \hat{\sigma}_{Y, n}^{(1)} \wedge\left((n+1)^{-1} s_{Y, n+1}^{\wedge} \rho_{Y}\right) .
$$

Thus, in view of equivalence (4.16) from Theorem 4.8 and Lemma 4.6, one has

$$
\hat{\sigma}_{Y, n}^{(2)}+\hat{\sigma}_{Y, n}^{(2)}\left(I \otimes\left(s_{Y, n+1}^{\wedge} \rho_{Y}\right)\right) A_{d \ell_{Y}}^{(2)} \approx 2 \frac{n}{n+1} \hat{\sigma}_{Y, n}^{(1)} \wedge\left(\hat{\sigma}_{Y, n}^{(1)}\left(I+s_{Y, n+1}^{\wedge} \rho_{Y}\right)\right) .
$$

Furthermore, assumption (4.22) implies that the trace norms of the operators on the r.h.s of (4.31) are uniformly bounded. Therefore, according to Remark 4.4,

$$
\left(\hat{\sigma}_{Y, n}^{(2)}-2 \hat{\sigma}_{Y, n}^{(1)} \wedge \hat{\sigma}_{Y, n}^{(1)}\right)\left(I \otimes\left(I+s_{Y, n+1}^{\wedge} \rho_{Y}\right)\right) A_{\mathscr{d}_{Y}}^{(2)} \approx 0 .
$$


The explicit form of $\hat{\sigma}_{Y, n}^{(2)}, \hat{\sigma}_{Y, n}^{(1)} \wedge \hat{\sigma}_{Y, n}^{(1)}$ given by Theorem 3.4 implies that $\hat{\sigma}_{Y, n}^{(2)}-2 \hat{\sigma}_{Y, n}^{(1)} \wedge \hat{\sigma}_{Y, n}^{(1)}$ and $\left(I \otimes\left(I+s_{Y, n+1}^{\wedge} \rho_{Y}\right)\right) A_{\mathscr{d}_{Y}}^{(2)}$ commute, which proves the self-adjointness of the operator on the 1.h.s of (4.32). Thus conditions (4.32), (4.27) for $q=2$, (4.28), and Lemma 4.5 yield relation (4.23) for $k=2$.

$\left(2^{\circ}\right)$ Assuming validity of equivalence (4.23) for $k \in\{2, \ldots, q-1\}$, where $q \in \mathbb{N}, q>2$, its validity will be proved for $k=q$.

By Theorem 3.1 for $n \geq q$ one has

$$
\begin{aligned}
\frac{1}{(n+1)^{q}}\left(\begin{array}{c}
n+1 \\
q
\end{array}\right) \hat{\sigma}_{Y, n+1}^{(q)}= & \frac{1}{(n+1)^{q-1}}\left(\begin{array}{c}
n \\
q-1
\end{array}\right) \hat{\sigma}_{Y, n}^{(q-1)} \wedge\left((n+1)^{-1} s_{Y, n+1}^{\wedge} \rho_{Y}\right) \\
& -\frac{1}{(n+1)^{q}}\left(\begin{array}{l}
n \\
q
\end{array}\right) \hat{\sigma}_{Y, n}^{(q)}\left(I^{\otimes(q-1)} \otimes\left(s_{Y, n+1}^{\wedge} \rho_{Y}\right)\right) A_{\mathscr{d}_{Y}}^{(q)}
\end{aligned}
$$

Assumption (4.22) implies

$$
\operatorname{Tr}\left|\hat{\sigma}_{Y, n}^{(q)}\left(I^{\otimes(q-1)} \otimes\left(s_{Y, n+1}^{\wedge} \rho_{Y}\right)\right) A_{\mathscr{d}_{Y}}^{(q)}\right| \leq s_{Y, n+1}^{\wedge}\left\|\rho_{Y}\right\| \quad \operatorname{Tr}\left|\hat{\sigma}_{Y, n}^{(q)}\right| \leq 2,
$$

hence, in view of (4.33), Remark 4.4, and the assumption $\hat{\sigma}_{Y, n}^{(q)} \approx \hat{\sigma}_{Y, n+1}^{(q)}$,

$$
\hat{\sigma}_{Y, n}^{(q)}+\hat{\sigma}_{Y, n}^{(q)}\left(I^{\otimes(q-1)} \otimes\left(s_{Y, n+1}^{\wedge} \rho_{Y}\right)\right) A_{\mathscr{\ell}_{Y}}^{(q)} \approx \frac{q !}{(n+1)^{q-1}}\left(\begin{array}{c}
n \\
q-1
\end{array}\right) \hat{\sigma}_{Y, n}^{(q-1)} \wedge\left((n+1)^{-1} s_{Y, n+1}^{\wedge} \rho_{Y}\right) .
$$

Thus, by relation (4.16) from Theorem 4.8, Lemma 4.6, and Remark 4.4, one has

$$
\hat{\sigma}_{Y, n}^{(q)}+\hat{\sigma}_{Y, n}^{(q)}\left(I^{\otimes(q-1)} \otimes\left(s_{Y, n+1}^{\wedge} \rho_{Y}\right)\right) A_{\mathscr{L}_{Y}}^{(q)} \approx \frac{q !}{(q-1) !} \hat{\sigma}_{Y, n}^{(q-1)} \wedge\left(\hat{\sigma}_{Y, n}^{(1)}\left(I+s_{Y, n+1}^{\wedge} \rho_{Y}\right)\right),
$$

since the trace norms of the operators on the r.h.s. of (4.36) are uniformly bounded, by assumption (4.22). Furthermore, in view of Lemma 4.6 and the inductive hypothesis $\hat{\sigma}_{Y, n}^{(q-1)} \approx$ $(q-1) ! \underbrace{\hat{\sigma}_{Y, n}^{(1)} \wedge \cdots \wedge \hat{\sigma}_{Y, n}^{(1)}}_{q-1}$, condition (4.36) yields

$$
\hat{\sigma}_{Y, n}^{(q)}+\hat{\sigma}_{Y, n}^{(q)}\left(I^{\otimes(q-1)} \otimes\left(s_{Y, n+1}^{\wedge} \rho_{Y}\right)\right) A_{\mathscr{d l}_{Y}}^{(q)} \approx q ! \underbrace{\hat{\sigma}_{Y, n}^{(1)} \wedge \cdots \wedge \hat{\sigma}_{Y, n}^{(1)}}_{q-1} \wedge\left(\hat{\sigma}_{Y, n}^{(1)}\left(I+s_{Y, n+1}^{\wedge} \rho_{Y}\right)\right),
$$


hence

$$
(\hat{\sigma}_{Y, n}^{(q)}-q ! \underbrace{\wedge_{\sigma_{Y, n}}^{(1)} \wedge \cdots \wedge \hat{\sigma}_{Y, n}^{(1)}}_{q})\left(I^{\otimes(q-1)} \otimes\left(I+s_{Y, n+1}^{\wedge} \rho_{Y}\right)\right) A_{\mathscr{d l}_{Y}}^{(q)} \approx 0 .
$$

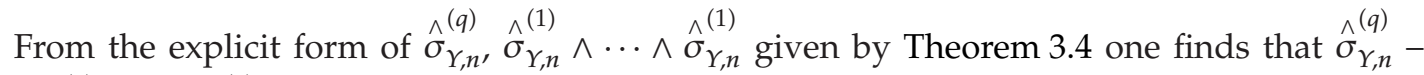
$q ! \hat{\sigma}_{Y, n}^{(1)} \wedge \cdots \wedge \hat{\sigma}_{Y, n}^{(1)}$ and $\left(I^{\otimes(q-1)} \otimes\left(I+s_{Y, n+1}^{\wedge} \rho_{Y}\right)\right) A_{\mathscr{d}_{Y}}^{(q)}$ commute, which proves the self-adjointness of the operator on the 1.h.s of (4.38). Thus conditions (4.38), (4.27), (4.28), and Lemma 4.5 yield $\hat{\sigma}_{Y, n}^{(q)} \approx q ! \underbrace{\wedge_{Y, n}^{(1)} \wedge \cdots \wedge \hat{\sigma}_{Y, n}^{(1)}}_{q}$. Validity of relation (4.23) has been proved.

Now turn to equivalence (4.25). Similarly to (4.27) one has

$$
\begin{aligned}
& \left(2-\left\|I+s_{Y, n+1}^{\vee} \rho_{Y}\right\|\right) \operatorname{Tr}\left|\begin{array}{l}
\vee^{(q)} \\
\sigma_{Y, n}
\end{array}-q ! \sigma_{Y, n}^{\vee^{(1)}} \vee \cdots \vee \frac{\vee^{(1)}}{\sigma_{Y, n}}\right| \\
& \leq \operatorname{Tr}\left|\left(\begin{array}{l}
\vee^{(q)} \\
\sigma_{Y, n}
\end{array}-q ! \sigma_{Y, n}^{\vee(1)} \vee \cdots \vee \underset{\sigma_{Y, n}}{\vee^{(1)}}\right)\left(I^{\otimes(q-1)} \otimes\left(I-s_{Y, n+1}^{\vee} \rho_{Y}\right)\right) S_{d d_{Y}}^{(q)}\right| .
\end{aligned}
$$

Furthermore, according to assumption (4.24),

$$
2-\left\|I+s_{Y, n+1}^{\vee} \rho_{Y}\right\| \geq 2-\left(1+s_{Y, n+1}^{\vee}\left\|\rho_{Y}\right\|\right) \geq 1-\epsilon>0 .
$$

The rest of the proof of (4.25) is by induction with respect to $k \geq 2$ and proceeds analogously to the proof of (4.23) with condition (4.28) replaced by (4.40) and the operators $I \mp s_{Y, n+1}^{\wedge} \rho_{Y}$ replaced by $I \pm s_{Y, n+1}^{\vee} \rho_{Y}$ (inversion of signs).

Theorem 4.9 allows to replace $(n, k)$-contractions of antisymmetric and symmetric product density operators by antisymmetric and symmetric products of 1-particle contractions, respectively, if the number $n$ of particles in the system is large. Further simplification, consisting in replacement of antisymmetric and symmetric products by tensor products, will be now proved possible. To this end weaker conditions on the asymptotic equivalence relation will be imposed.

Definition 4.10. Fix $k \in \mathbb{N}$ and $d \in \mathbb{R}, d>0$. Families $\left\{A_{Y, n}\right\}_{(Y, n) \in \mathcal{M}(\Omega) \times \mathbb{N}},\left\{B_{Y, n}\right\}_{(Y, n) \in \mathcal{M}(\Omega) \times \mathbb{N}}$ of operators $A_{Y, n}, B_{Y, n} \in \tau\left(\mathscr{\ell}_{Y}^{\otimes k}\right)$ are called weakly asymptotically equivalent (symbolically: $A_{Y, n} \sim$ $\left.B_{Y, n}\right)$, if $d-\lim _{n, \mu(Y) \rightarrow \infty} \operatorname{Tr}\left(A_{Y, n}-B_{Y, n}\right) C_{Y, n}=0$ for every family $\left\{C_{Y, n}\right\}_{(Y, n) \in \mathcal{M}(\Omega) \times \mathbb{N}}$ of operators of the form $C_{Y, n}=\bigotimes_{i=1}^{k} C_{Y, n}^{(i)}$, where $C_{Y, n}^{(i)} \in \mathcal{B}\left(\mathscr{L}_{Y}\right)(i \in\{1, \ldots, k\},(Y, n) \in \mathcal{M}(\Omega) \times \mathbb{N})$ are operators with uniformly bounded operator norms.

\section{Remark 4.4.}

The relation $\sim$ has the properties analogous to the properties of the relation $\approx$ from

Definition 4.11. Let $k \in \mathbb{N}, k \geq 2$. Fix $\pi \in S_{k}$. A set $X \subset\{1, \ldots, k\}$ is called a cyclic set of the permutation $\pi$, if $X=\left\{l_{1}, \ldots, l_{q}\right\}$ for some $l_{1}, \ldots, l_{q} \in\{1, \ldots, k\}, q \in\{2, \ldots, k\}$, such that 
$\pi\left(l_{s}\right)=l_{s+1}$ for every $s \in\{1, \ldots, q-1\}$, and $\pi\left(l_{q}\right)=l_{1}$. A singleton $\{l\} \subset\{1, \ldots, k\}$ such that $\pi(l)=l$ is also called a cyclic set of the permutation $\pi$.

Note that the set $\{1, \ldots, k\}$ from the above definition can be represented as the union of disjoint cyclic sets of $\pi$.

Lemma 4.12. Let $k \in \mathbb{N}, k \geq 2$. If $B^{(1)}, \ldots, B^{(k)} \in \mathcal{\tau}\left(\mathscr{H}_{Y}\right)$ then

$$
k ! \operatorname{Tr}\left(B_{Y, n}^{(1)} \otimes \cdots \otimes B_{Y, n}^{(k)}\right) A_{\mathscr{\ell}_{Y}}^{(k)}=\sum_{\pi \in S_{k}} \operatorname{sgn} \pi \prod_{j=1}^{p(\pi)} \operatorname{Tr} \prod_{s=1}^{q_{j}} B_{Y, n}^{\left(l_{j, s}\right)},
$$

where $p(\pi) \in\{1, \ldots, k\}$ is the number of disjoint cyclic sets of $\pi$, indexed by $j$, and $q_{j}$ denotes the number of elements of the $j$ th cyclic set of $\pi$, which is $\left\{l_{j, 1}, \ldots, l_{j, q_{j}}\right\}$, where

$$
\pi\left(l_{j, q_{j}}\right)=l_{j, 1}, \quad \text { for } q_{j} \geq 2, \quad \pi\left(l_{j, s}\right)=l_{j, s+1}, \quad s=1, \ldots, q_{j}-1
$$

Clearly, $\sum_{j=1}^{p(\pi)} q_{j}=k$ and $\bigcup_{j=1}^{p(\pi)} \bigcup_{s=1}^{q_{j}}\left\{l_{j, s}\right\}=\{1, \ldots, k\}$.

Proof. Let $\left\{\varphi_{i}\right\}_{i \in \mathbb{N}}$ be an orthonormal basis of $\mathscr{\ell}_{Y}$. One has

$$
k ! \operatorname{Tr}\left(B_{Y, n}^{(1)} \otimes \cdots \otimes B_{Y, n}^{(k)}\right) A_{\mathscr{d}_{Y}}^{(k)}=\sum_{\pi \in S_{k}} \operatorname{sgn} \pi \prod_{j=1}^{p(\pi)} M_{j},
$$

where

$$
M_{j}:=\sum_{i_{j, 1}=1}^{\infty} \ldots \sum_{i_{l_{j, q_{j}}}=1}^{\infty}\left\langle\varphi_{i_{l_{j, 1}}} B^{\left(l_{j, 1}\right)} \varphi_{\left.i_{\pi\left(l_{j, 1}\right)}\right)}\right\rangle \cdots\left\langle\varphi_{i_{l_{j, q_{j}}}} B^{\left(l_{j, q_{j}}\right)} \varphi_{i_{\pi\left(l_{j, q_{j}}\right)}}\right\rangle .
$$

If $q_{j}>2$ for some $j \in\{1, \ldots, p(\pi)\}$ then, by condition (4.42) and Parseval's formula,

$$
\begin{aligned}
& M_{j}=\sum_{i_{l_{j, 1}}=1}^{\infty} \ldots \sum_{i_{l_{j, q_{j}}}=1}^{\infty}\left\langle\varphi_{i_{i_{j, 1}}} B^{\left(l_{j, 1}\right)} \varphi_{i_{l_{j, 2}}}\right\rangle\left\langle\varphi_{i_{l_{j, 2}}} B^{\left(l_{j, 2}\right)} \varphi_{i_{l_{j, 3}}}\right\rangle \cdots\left\langle\varphi_{i_{l_{j, q_{j}}}} B^{\left(l_{j, q_{j}}\right)} \varphi_{i_{l_{j, 1}}}\right\rangle \\
& =\sum_{i_{j, 1}=1}^{\infty} \sum_{i_{j, 3}=1}^{\infty} \ldots \sum_{i_{j, q_{j}}=1}^{\infty}\left\langle\varphi_{i_{l_{j, 1}}} B^{\left(l_{j, 1}\right)} B^{\left(l_{j, 2}\right)} \varphi_{i_{l_{j, 3}}}\right\rangle \cdots\left\langle\varphi_{i_{l_{j, q_{j}}}} B^{\left(l_{j, q_{j}}\right)} \varphi_{i_{l_{j, 1}}}\right\rangle .
\end{aligned}
$$

Performing successive summations one then obtains

$$
M_{j}=\sum_{i_{l_{j, 1}}=1}^{\infty}\left\langle\varphi_{i_{l_{j, 1}}},\left(\prod_{s=1}^{q_{j}} B^{\left(l_{j, s}\right)}\right) \varphi_{i_{l_{j, 1}}}\right\rangle=\operatorname{Tr} \prod_{s=1}^{q_{j}} B^{\left(l_{j, s}\right)}
$$


The derivation of the above formula for $q_{j}=1,2$, after simplifications, proceeds analogously. This completes the proof of (4.41), in view of (4.43).

Lemma 4.13. One has

$$
\underset{n, \mu(Y) \rightarrow \infty}{d-\lim _{n \rightarrow, n}}\left\|\hat{\sigma}_{Y, n}^{(1)}\right\|=0
$$

and if $\stackrel{\vee^{(2)}}{\sigma_{Y, n}} \approx 2 \stackrel{\vee^{(1)}}{\sigma_{Y, n}} \vee \stackrel{\vee}{\sigma}_{Y, n}^{(1)}$ (see Theorem 4.9) then

$$
\underset{n, \mu(Y) \rightarrow \infty}{d-\lim _{n \rightarrow, n}}\left\|\begin{array}{c}
v^{(1)} \\
\sigma_{Y, n}
\end{array}\right\|=0
$$

Proof. To prove (4.47) it suffices to observe that, according to Theorem 2.3,

$$
\left\|\hat{\sigma}_{Y, n}^{(1)}\right\|=\left\|\mathrm{L}_{n}^{1}\left(\frac{1}{\xi_{Y, n}^{\wedge}} \rho_{Y}^{\wedge n}\right)\right\| \leq \frac{1}{n} \frac{1}{\xi_{Y, n}^{\wedge}}\left\|\rho_{Y}^{\wedge n}\right\| \leq \frac{1}{n} \frac{1}{\xi_{Y, n}^{\wedge}} \operatorname{Tr} \rho_{Y}^{\wedge n}=\frac{1}{n} .
$$

Now (4.48) will be proved. Let $\left\{\varphi_{i}\right\}_{i \in \mathbb{N}}$ be an orthonormal basis of $\mathfrak{L}_{Y}$ for fixed $Y \in$ $\mathcal{M}(\Omega)$. Then

$$
\begin{aligned}
& \operatorname{Tr} 2 \stackrel{\vee^{(1)}}{\sigma_{Y, n}} \vee \stackrel{\vee^{(1)}}{\sigma_{Y, n}}=2 \operatorname{Tr}\left(\stackrel{\vee^{(1)}}{\sigma_{Y, n}} \otimes \stackrel{\vee^{(1)}}{\sigma_{Y, n}}\right) S_{d^{(2)}}^{(2)} \\
& =\sum_{\pi \in S_{2}} \sum_{i_{1}, i_{2}=1}^{\infty}\left\langle\varphi_{i_{1}}, \sigma_{Y, n}^{\vee(1)} \varphi_{i_{\pi(1)}}\right\rangle\left\langle\varphi_{i_{2}}, \sigma_{Y, n}^{\vee(1)} \varphi_{i_{\pi(2)}}\right\rangle \\
& =\left(\operatorname{Tr} \stackrel{\vee(1)}{\sigma_{Y, n}^{(1)}}\right)^{2}+\operatorname{Tr}\left(\stackrel{\vee}{\stackrel{v}{\sigma}_{Y, n}^{(1)}} \underset{\sigma_{Y, n}^{(1)}}{\sigma^{(1)}}\right) .
\end{aligned}
$$

Taking into account (4.50), the relation $\stackrel{v_{Y, n}^{(2)}}{\sigma_{Y}} \approx 2 \stackrel{\vee_{Y, n}^{(1)}}{\sigma_{Y, n}} \stackrel{\vee^{(1)}}{\sigma_{Y, n}}$, Definition 4.2 for $C_{Y, n}=I^{\otimes 2}$, and the equality $\operatorname{Tr} \stackrel{\vee^{(2)}}{\sigma_{Y, n}}=\operatorname{Tr} \stackrel{\vee^{(1)}}{\sigma_{Y, n}}=1$, one obtains

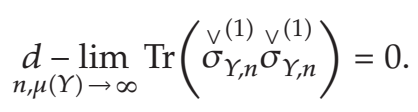

Furthermore,

$$
\left\|\begin{array}{l}
\vee^{(1)} \\
\sigma_{Y, n} \varphi
\end{array}\right\|^{2}=\left\langle\varphi, \stackrel{\vee^{(1)}}{\sigma_{Y, n}^{(1)}} \sigma_{Y, n} \varphi\right\rangle \leq \operatorname{Tr}\left(\begin{array}{c}
\vee^{(1)} \vee^{(1)} \\
\sigma_{Y, n} \sigma_{Y, n}
\end{array}\right)
$$

for every $\varphi \in \mathscr{L}_{\Upsilon}$ such that $\|\varphi\|=1$, hence (4.51) yields (4.48).

Notice that (4.47) can be also proved analogously to (4.48) under the additional assumption $\hat{\sigma}_{Y, n}^{(2)} \approx 2 \hat{\sigma}_{Y, n}^{(1)} \wedge \hat{\sigma}_{Y, n}^{(1)}$. 
The proof of the next theorem for $k=2$ was given in [11, 12].

Theorem 4.14 (Asymptotic formulae II). Let $k \in \mathbb{N}, k \geq 2$. One has

$$
k ! \underbrace{\hat{\sigma}_{Y, n}^{(1)} \wedge \cdots \wedge \hat{\sigma}_{Y, n}^{(1)}}_{k} \sim \underbrace{\hat{\sigma}_{Y, n}^{(1)} \otimes \cdots \otimes \hat{\sigma}_{Y, n}^{(1)}}_{k}
$$

and if $d-\lim _{n, \mu(Y) \rightarrow \infty}\left\|\sigma_{Y, n}^{(1)}\right\|=0$ (see Lemma 4.13) then

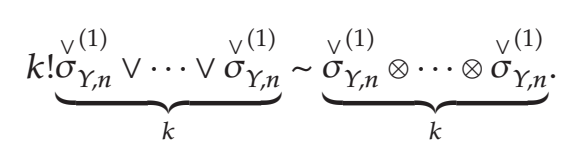

Proof. First (4.53) will be proved. Fix a family $\left\{C_{Y, n}\right\}_{(Y, n) \in \mathcal{M}(\Omega) \times \mathbb{N}}$ of operators such as in Definition 4.10 and set

$$
B_{Y, n}^{(r)}:=\hat{\sigma}_{Y, n}^{(1)} C_{Y, n^{\prime}}^{(r)} \quad r=1, \ldots, k
$$

Then, by Lemma 4.12, one has

$$
\begin{aligned}
\operatorname{Tr} k ! & \left(\hat{\sigma}_{Y, n}^{(1)} \wedge \cdots \wedge \hat{\sigma}_{Y, n}^{(1)}\right)\left(C_{Y, n}^{(1)} \otimes \cdots \otimes C_{Y, n}^{(k)}\right) \\
= & k ! \operatorname{Tr}\left(B_{Y, n}^{(1)} \otimes \cdots \otimes B_{Y, n}^{(k)}\right) A_{\mathscr{C}_{Y}}^{(k)}=\sum_{\pi \in S_{k}} \operatorname{sgn} \pi \prod_{j=1}^{p(\pi)} \operatorname{Tr} \prod_{s=1}^{q_{j}} B_{Y, n}^{\left(l_{j, s}\right)} \\
= & \operatorname{Tr}\left(\hat{\sigma}_{Y, n}^{(1)} \otimes \cdots \otimes \hat{\sigma}_{Y, n}^{(1)}\right)\left(C_{Y, n}^{(1)} \otimes \cdots \otimes C_{Y, n}^{(k)}\right) \\
& +\sum_{\substack{\pi \in S_{k} \\
\pi \neq \mathrm{Id}}} \operatorname{sgn} \pi \prod_{j=1}^{p(\pi)} \operatorname{Tr} \prod_{s=1}^{q_{j}} B_{Y, n}^{\left(l_{j, s}\right)} .
\end{aligned}
$$

Thus

$$
\begin{gathered}
\operatorname{Tr}\left(k ! \hat{\sigma}_{Y, n}^{(1)} \wedge \cdots \wedge \hat{\sigma}_{Y, n}^{(1)}-\hat{\sigma}_{Y, n}^{(1)} \otimes \cdots \otimes \hat{\sigma}_{Y, n}^{(1)}\right)\left(C_{Y, n}^{(1)} \otimes \cdots \otimes C_{Y, n}^{(k)}\right) \\
=\sum_{\substack{\pi \in S_{k} \\
\pi \neq \mathrm{Id}}} \operatorname{sgn} \pi \prod_{j=1}^{p(\pi)} \operatorname{Tr} \prod_{s=1}^{q_{j}} B_{Y, n}^{\left(l_{j, s}\right)} .
\end{gathered}
$$


Now, let $\pi \in S_{k}, \pi \neq \mathrm{Id}$, be fixed. If $q_{j}=1$ for some $j \in\{1, \ldots, p(\pi)\}$ then

$$
\left|\operatorname{Tr} \prod_{s=1}^{q_{j}} B_{Y, n}^{\left(l_{j, s}\right)}\right| \equiv\left|\operatorname{Tr} B_{Y, n}^{\left(l_{j, 1}\right)}\right| \leq\left\|C_{Y, n}^{\left(l_{j, 1}\right)}\right\| \quad \operatorname{Tr}\left|\hat{\sigma}_{Y, n}^{(1)}\right|=\left\|C_{Y, n}^{\left(l_{j, 1}\right)}\right\|,
$$

whereas if $q_{j} \geq 2$ then

$$
\begin{aligned}
\left|\operatorname{Tr} \prod_{s=1}^{q_{j}} B_{Y, n}^{\left(l_{j, s}\right)}\right| & \leq\left\|\prod_{s=1}^{q_{j}-1} B_{Y, n}^{\left(l_{j, s}\right)}\right\| \operatorname{Tr}\left|B_{Y, n}^{\left(l_{j, q_{j}}\right)}\right| \\
& \leq\left\|\hat{\sigma}_{Y, n}^{(1)}\right\|^{q_{j}-1}\left(\prod_{s=1}^{q_{j}-1}\left\|C_{Y, n}^{\left(l_{j, s}\right)}\right\|\right)\left\|C_{Y, n}^{\left(l_{j, q_{j}}\right)}\right\| \operatorname{Tr}\left|\hat{\sigma}_{Y, n}^{(1)}\right| \\
& \leq\left\|\hat{\sigma}_{Y, n}^{(1)}\right\|^{q_{j}-1} \prod_{s=1}^{q_{j}}\left\|C_{Y, n}^{\left(l_{j, s}\right)}\right\| .
\end{aligned}
$$

Since $\pi \neq \mathrm{Id}$, there exists at least one $j \in\{1, \ldots, p(\pi)\}$ such that $q_{j} \geq 2$, hence

$$
\begin{aligned}
\left|\prod_{j=1}^{p(\pi)} \operatorname{Tr} \prod_{s=1}^{q_{j}} B_{Y, n}^{\left(l_{j, s}\right)}\right| & \leq\left(\prod_{j=1}^{p(\pi)}\left\|\hat{\sigma}_{Y, n}^{(1)}\right\|^{q_{j}-1}\right) \prod_{j=1}^{p(\pi)} \prod_{s=1}^{q_{j}}\left\|C_{Y, n}^{\left(l_{j, s}\right)}\right\| \\
& =\left\|C_{Y, n}\right\| \prod_{j=1}^{p(\pi)}\left\|\hat{\sigma}_{Y, n}^{(1)}\right\|^{q_{j}-1}
\end{aligned}
$$

and at least one exponent $q_{j}-1$ is nonzero. Thus, by the uniform boundedness of the norms $\left\|C_{Y, n}\right\|$ and Lemma 4.13 , the termodynamic limit of the 1.h.s of (4.57) equals 0 , which proves the validity of relation (4.53).

The proof of relation (4.54), after discarding the permutation signs and replacing $\wedge$ by $\checkmark$, proceeds analogously.

\section{Appendix}

\section{Product Integral Kernels of Trace Class Operators}

In this section theorems concerning product integral kernels, exploited in Section 3, are formulated.

Fix the Hilbert space $\mathscr{\ell}_{Y}:=L^{2}(Y, \mu)$ over the field $\mathbb{K}=\mathbb{C}$ or $\mathbb{R}$, where the measure $\mu$ is separable and $\sigma$-finite. For every $n \in \mathbb{N}$ the space $\mathfrak{l}_{Y}^{\otimes n}$ is identified with $L^{2}\left(Y^{n}, \mu^{\otimes n}\right)$. Unless otherwise stated, elements of $L^{2}$ spaces are identified with their representatives and denoted by the same symbols. 
Let $\mathcal{K} \in L^{2}\left(Y^{2}, \mu^{\otimes 2}\right)$. In the case of the integral operator $K: H_{Y} \rightarrow H_{Y}$ defined for every $\varphi \in H_{Y}$ and $\mu$-a.a. $x \in Y$ by

$$
(K \varphi)(x)=\int_{Y} \mathcal{K}(x, y) \varphi(y) \mathrm{d} \mu(y)
$$

both $\mathcal{K}$ regarded as an element of $L^{2}\left(Y^{2}, \mu^{\otimes 2}\right)$ as well as its arbitrary representative is called an integral kernel of $K$. The kernel $\mathcal{K}$ is unique as an element of $L^{2}\left(Y^{2}, \mu^{\otimes 2}\right)$ but a representative of $\mathcal{K}$ of a special form, given in Lemma A.3 and Definition A.4, is useful in computations of the trace of $K$.

Let $\mathscr{L} S\left(\mathscr{\ell}_{Y}\right)$ be the space of Hilbert-Schmidt operators on $\mathfrak{l}_{Y}$ with the inner product defined by $\langle A, B\rangle_{\mathscr{H} S\left(\mathscr{d}_{Y}\right)}:=\operatorname{Tr} A^{*} B$ and the induced norm denoted by $\|\cdot\|_{\mathscr{d}\left(\mathscr{d}_{Y}\right)}$. In the sequel use is made of the following theorem, the proof of which can be found in [19].

Theorem A.1. An operator $K \in \mathbb{B}\left(H_{Y}\right)$ is Hilbert-Schmidt if and only if it is an integral operator with an integral kernel $\mathcal{K} \in L^{2}\left(Y^{2}, \mu^{\otimes 2}\right)$. Furthermore, $\|K\|_{\mathscr{\ell} S\left(H_{Y}\right)}=\|\mathcal{K}\|_{L^{2}\left(Y^{2}, \mu^{\otimes 2}\right)}$.

Corollary A.2. Let $K, G \in \mathscr{H} \mathcal{S}\left(H_{Y}\right)$ and let $\mathcal{K}, \mathcal{G} \in L^{2}\left(Y^{2}, \mu^{\otimes 2}\right)$ be integral kernels of the operators $K, G$, respectively. Then $\langle K, G\rangle_{\mathscr{H} S\left(H_{Y}\right)}=\langle\mathcal{K}, \mathcal{G}\rangle_{L^{2}\left(Y^{2}, \mu^{\otimes 2}\right)}$.

Recall that $K \in \mathbb{B}\left(H_{Y}\right)$ is a trace class operator if and only if there exist operators $K_{1}, K_{2} \in \mathscr{H} \mathcal{S}\left(H_{Y}\right)$ such that $K=K_{1} K_{2}$. Moreover, $\operatorname{Tr} K=\left\langle K_{1}^{*}, K_{2}\right\rangle_{\mathscr{H} S\left(H_{Y}\right)}$. This fact, Theorem A.1, and Corollary A.2 imply the following lemma, in which elements of the $L^{2}$ space are distinguished from their representatives. The element of the $L^{2}$ space represented by a function $f$ is denoted by $[f]$.

Lemma A.3. Let $K \in \boldsymbol{\tau}\left(H_{Y}\right), K=K_{1} K_{2}$, where $K_{1}, K_{2} \in \operatorname{deS}\left(H_{Y}\right)$. Let [ $\left.\mathcal{K}_{1}\right],\left[\mathcal{K}_{2}\right] \in$ $L^{2}\left(Y^{2}, \mu^{\otimes 2}\right)$ be integral kernels of $K_{1}, K_{2}$. Then for any choice of representatives $\mathcal{K}_{1} \in\left[\mathcal{K}_{1}\right]$, $\mathcal{K}_{2} \in\left[\mathcal{K}_{2}\right]$ the function $\mathcal{K}: Y \times Y \rightarrow \mathbb{K}$ defined for $\mu^{\otimes 2}-a . a .(x, y) \in Y \times Y$ by

$$
\mathcal{K}(x, y)=\int_{Y} \mathcal{K}_{1}(x, z) \mathcal{K}_{2}(z, y) \mathrm{d} \mu(z)
$$

is $\mu^{\otimes 2}$-square integrable and it is an integral kernel of $K$. The function $\mathcal{L}: Y \rightarrow \mathbb{K}$ defined for $\mu$-a.a. $x \in Y$ by $\mathcal{L}(x)=\mathcal{K}(x, x)$ is $\mu$-integrable. Moreover,

$$
\operatorname{Tr} K=\int_{Y} \mathcal{L}(x) \mathrm{d} \mu(x) \equiv \int_{Y} \mathcal{K}(x, x) \mathrm{d} \mu(x) .
$$

Definition A.4. Under the assumptions of Lemma A.3, the function $\mathcal{K}$ given by formula (A.2) (for any choice of representatives $\mathcal{K}_{1}, \mathcal{K}_{2}$ of $\left[\mathcal{K}_{1}\right],\left[\mathcal{K}_{2}\right]$ ) is called a product integral kernel of $K$.

Notice that for $\mu$ being the Lebesgue measure on $[0,1] \times[0,1]$ formula (A.3) is valid, for example, if $\mathcal{K}$ is any continuous function.

In the following lemma, which follows from Lemma A.3, the function $\mathcal{K}_{0}$ need not be a product integral kernel of $K_{0}$ but the integral formula for the trace of $K_{0}$ still holds for $\mathcal{K}_{0}$. 
Lemma A.5. Let $k, n \in \mathbb{N}, k<n$, and let $\mathcal{K}$ be a product integral kernel of $K \in \mathcal{\tau}\left(H_{Y}^{\otimes n}\right) \equiv$ $\tau\left(L^{2}\left(Y^{n}, \mu^{\otimes n}\right)\right)$. Then the function $\mathcal{K}_{0}: Y^{k} \times Y^{k} \rightarrow \mathbb{K}$ defined for $\mu^{\otimes 2 k}$-a.a. $\left(x^{\prime}, y^{\prime}\right) \in Y^{k} \times Y^{k}$ by

$$
\mathcal{K}_{0}\left(x^{\prime}, y^{\prime}\right)=\int_{Y^{n-k}} \mathcal{K}\left(x^{\prime}, x^{\prime \prime}, y^{\prime}, x^{\prime \prime}\right) \mathrm{d} \mu^{\otimes(n-k)}\left(x^{\prime \prime}\right)
$$

is $\mu^{\otimes 2 k}$-square integrable and the integral operator $K_{0}$ with the kernel $\mathcal{K}_{0}$ belongs to $\tau\left(H_{\gamma}^{\otimes k}\right)$. For every $x, \varphi \in H_{Y}^{\otimes k}$ and every orthonormal basis $\left\{\psi_{i}\right\}_{i \in \mathbb{N}}$ of $H_{Y}^{\otimes(n-k)}$ one has

$$
\left\langle\chi, K_{0} \varphi\right\rangle_{H_{Y}^{\otimes k}}=\sum_{i=1}^{\infty}\left\langle\chi \otimes \psi_{i}, K\left(\varphi \otimes \psi_{i}\right)\right\rangle_{H_{Y}^{\otimes n}}
$$

The function $\mathcal{L}_{0}: Y^{k} \rightarrow \mathbb{K}$ defined for $\mu^{\otimes k}$-a.a. $x^{\prime} \in Y^{k}$ by $\mathcal{L}_{0}\left(x^{\prime}\right)=\mathcal{K}_{0}\left(x^{\prime}, x^{\prime}\right)$ is $\mu^{\otimes k}$-integrable. Moreover,

$$
\int_{Y^{k}} \mathcal{K}_{0}\left(x^{\prime}, x^{\prime}\right) d \mu^{\otimes k}\left(x^{\prime}\right) \equiv \int_{Y^{k}} \mathcal{L}_{0}\left(x^{\prime}\right) \mathrm{d} \mu^{\otimes k}\left(x^{\prime}\right)=\operatorname{Tr} K_{0}=\operatorname{Tr} K
$$

Corollary A.6. Under the assumptions of Lemma A.5, if $C \in \mathcal{B}\left(H_{Y}^{\otimes k}\right)$ then $\operatorname{Tr} C K_{0}=\operatorname{Tr}(C \otimes$ $\left.I^{\otimes(n-k)}\right) K$.

\section{Acknowledgments}

This paper presents the results of a part of the author's MS thesis [2] written in the Institute of Physics, Nicolaus Copernicus University, Torun, under the supervision of Professor Jan Maćkowiak. The author wishes to express his gratitude to Professor Maćkowiak for helpful suggestions and remarks. Professor Maćkowiak prepared also, on his own initiative, the English translation of appropriate parts of the author's thesis, which was useful for the author in editing of the present paper.

\section{References}

[1] W. Radzki, "Contractions of product density operators of systems of identical fermions and bosons," arXiv:0809.0474v2, 2008, http:/ / arxiv.org/abs/0809.0474v2 .

[2] W. Radzki, Kummer contractions of product density matrices of systems of $n$ fermions and $n$ bosons, M.S. thesis, Institute of Physics, Nicolaus Copernicus University, Torun, Poland, 1999.

[3] J. von Neumann, Mathematische Grundlagen der Quantenmechanik, Springer, Berlin, Germany, 1932.

[4] H. Kummer, " $n$-representability problem for reduced density matrices," Journal of Mathematical Physics, vol. 8, pp. 2063-2081, 1967.

[5] A. J. Coleman, "Structure of fermion density matrices," Reviews of Modern Physics, vol. 35, no. 3, pp. $668-689,1963$.

[6] C. Garrod and J. K. Percus, "Reduction of the N-particle variational problem," Journal of Mathematical Physics, vol. 5, pp. 1756-1776, 1964.

[7] H. Kummer, "Mathematical description of a system consisting of identical quantum-mechanical particles," Journal of Mathematical Physics, vol. 11, pp. 449-474, 1970.

[8] "Reduced density operators with applications to physical and chemical systems. II," in Proceedings of a Conference held at Queen's University, Kingston, Ont., June 20-22, 1974, R. M. Erdahl, Ed., Queen's Papers on Pure and Applied Mathematics, no. 40, Queen's University, Kingston, Canada, 1974. 
[9] H. Grudziński and J. Hirsch, "Searching for new conditions for fermion N-representability," Reports on Mathematical Physics, vol. 53, no. 3, pp. 331-338, 2004.

[10] A. J. Coleman and V. I. Yukalov, Reduced Density Matrices. Coulson's Challenge, vol. 72 of Lecture Notes in Chemistry, Springer, Berlin, Germany, 2000.

[11] A. Kossakowski and J. Maćkowiak, "Minimization of the free energy of large continuous quantum systems over product states," Reports on Mathematical Physics, vol. 24, no. 3, pp. 365-376, 1986.

[12] J. Maćkowiak, "Infinite-volume limit of continuous n-particle quantum systems," Physics Reports, vol. 308, no. 4, pp. 235-331, 1999.

[13] J. Maćkowiak and M. Wiśniewski, "A perturbation expansion for the free energy of the $s-d$ exchange Hamiltonian," Physica A, vol. 242, no. 3-4, pp. 482-500, 1997.

[14] G. E. Volovik, Exotic Properties of Superfluid ${ }^{3}$ He, World Scientific, Signapore, 1992.

[15] P. Tarasewicz and J. Maćkowiak, "Thermodynamic functions of Fermi gas with quadruple BCS-type binding potential," Physica C, vol. 329, no. 2, pp. 130-148, 2000.

[16] J. Maćkowiak and P. Tarasewicz, "Extension of the Bardeen-Cooper-Schrieffer model of superconductivity," Physica C, vol. 331, no. 1, pp. 25-37, 2000.

[17] C. W. Schneider, G. Hammerl, G. Logvenov et al., "Half- $h / 2 e$ critical current-oscillations of SQUIDs," Europhysics Letters, vol. 68, no. 1, pp. 86-92, 2004.

[18] D. Ruelle, Statistical Mechanics: Rigorous Results, W. A. Benjamin, New York, NY, USA, 1969.

[19] R. Schatten, Norm Ideals of Completely Continuous Operators, vol. 27 of Ergebnisse der Mathematik und ihrer Grenzgebiete. N. F., Springer, Berlin, Germany, 1960. 


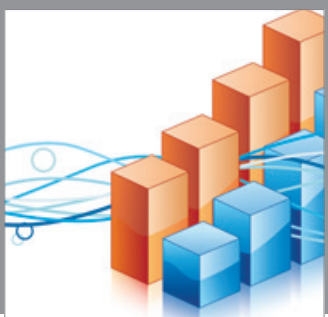

Advances in

Operations Research

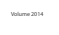

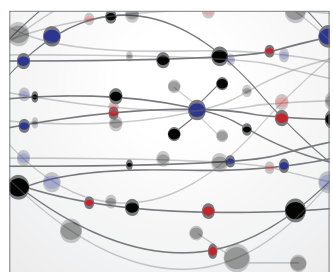

\section{The Scientific} World Journal
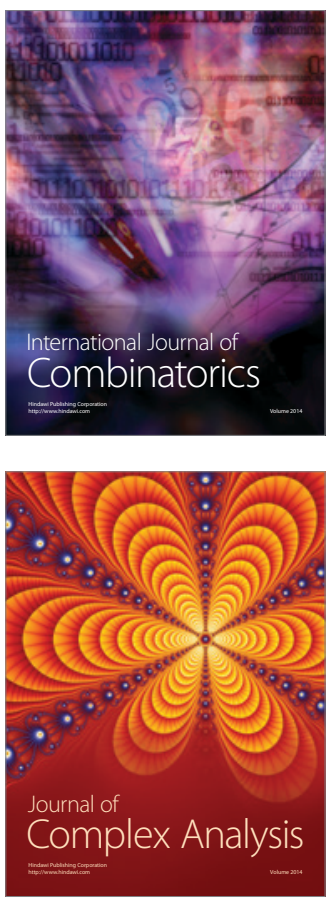

International Journal of

Mathematics and

Mathematical

Sciences
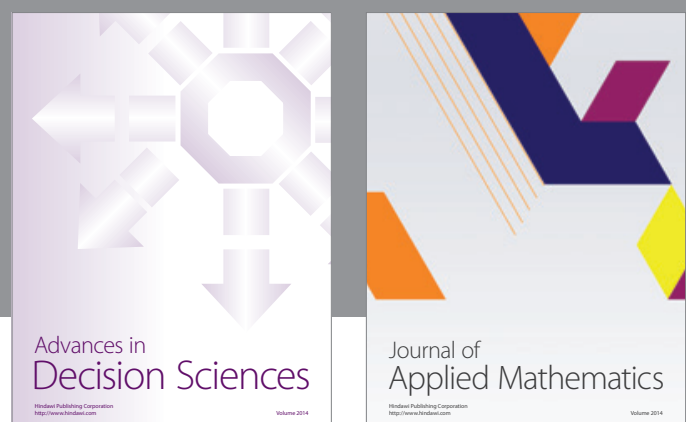

Journal of

Applied Mathematics
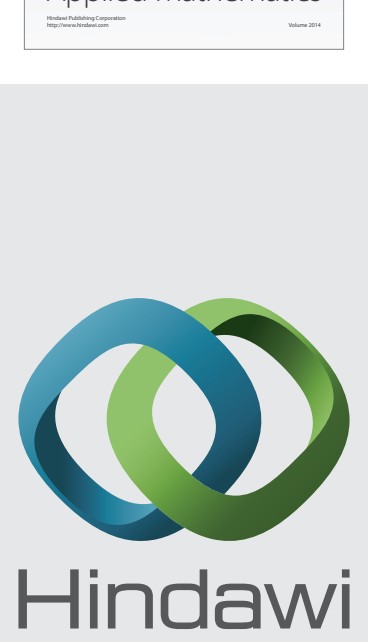

Submit your manuscripts at http://www.hindawi.com
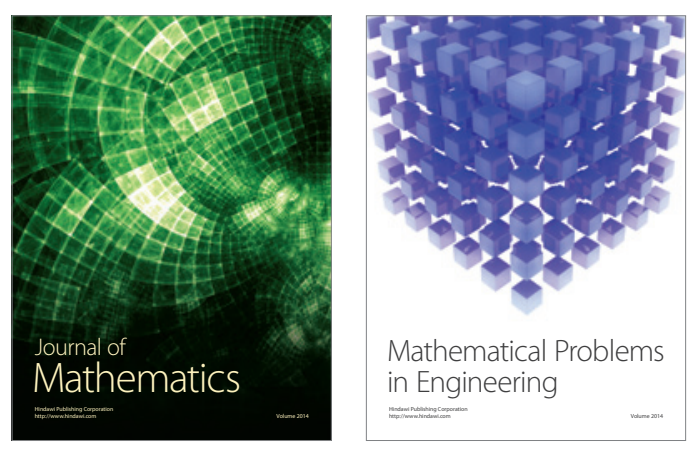

Mathematical Problems in Engineering
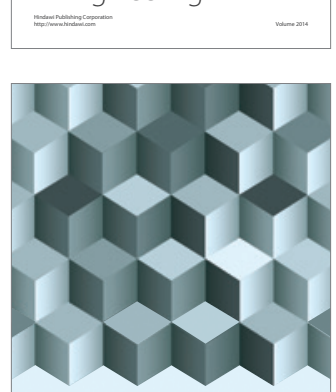

Journal of

Function Spaces
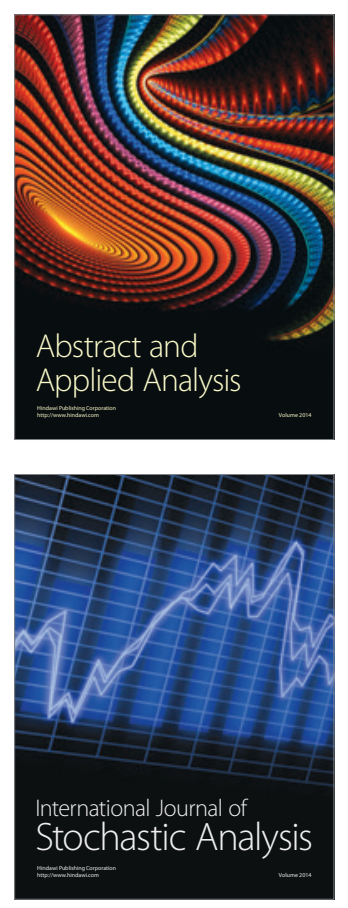

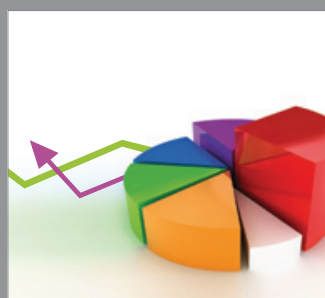

ournal of

Probability and Statistics

Promensencen
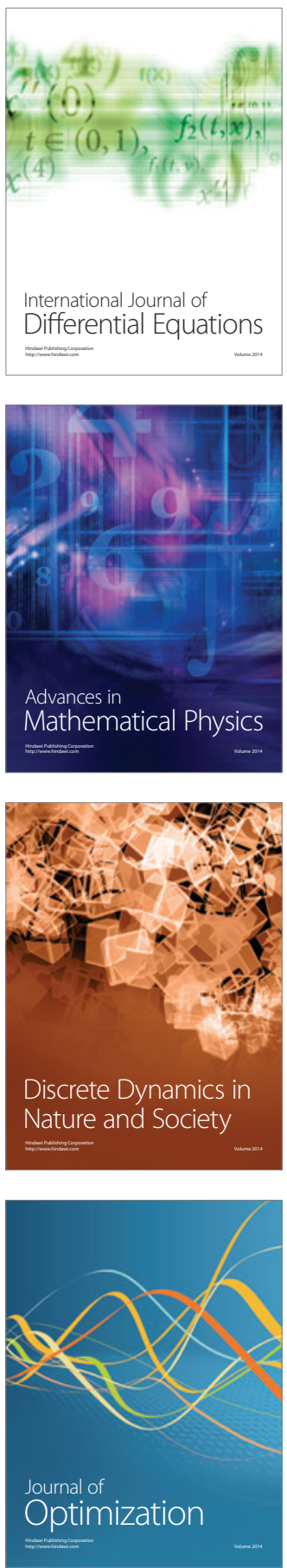